\title{
System-level performance of LTE-Advanced with joint transmission and dynamic point selection schemes
}

\author{
Helka-Liina Määttänen ${ }^{*}$, Kari Hämäläinen ${ }^{1}$, Juha Venäläinen ${ }^{2}$, Karol Schober ${ }^{3}$, \\ Mihai Enescu' and Mikko Valkama²
}

\begin{abstract}
In this article, we present a practical coordinated multipoint (COMP) system for LTE-Advanced. In this CoMP system, cooperation is enabled for cell-edge users via dynamic switching between the normal single-cell operation and CoMP. We first formulate a general CoMP system model of several CoMP schemes. We then investigate a practical finite-rate feedback design that simultaneously supports interference coordination, joint transmission (JT), and dynamic point selection (DPS) with a varying number of cooperating transmission points while operating a single-cell transmission as a fallback mode. We provide both link-level and system-level results for the evaluation of different feedback options for general CoMP operation. The results show that there are substantial performance gains in cell-edge throughputs for both JT and DPS CoMP over the baseline Release 10 LTE-Advanced with practical feedback options. We also show that CoMP can enable improved mobility management in real networks.
\end{abstract}

\section{Introduction}

Multiple-input multiple-output (MIMO) systems have the potential to provide the capacity needed for futuregeneration wireless systems, and for this reason they have been adopted by 3GPP Long-Term Evolution (LTE) and LTE-Advanced (LTE-A) [1,2]. MIMO operation was already defined in the early stage of LTE specification work. In the downlink, $2 \times 2$ and $4 \times 4$ MIMO operation have been defined in Release 8 [3], and these have been further extended to $8 \times 8 \mathrm{MIMO}$ in Release 10 [2]. The main scenario is single-user (SU)-MIMO, where spatial multiplexing within individual time-frequency resource blocks is performed for a single user equipment (UE) at a time. In addition, multi-user (MU)-MIMO operation, where a time-frequency resource block is shared by multiple users in the spatial domain, has been possible since Release 8. In LTE Release 8, MU-MIMO is allowed only in a standard non-transparent manner, but in LTE Release 9 and 10 it can be enabled in a standard transparent manner. In Release 10, certain features have been included to improve the MU-MIMO performance com-

\footnotetext{
*Correspondence: helka.maattanen@renesasmobile.com

1 Renesas Mobile Europe Ltd., Porkkalankatu 24, 00180 Helsinki, Finland Full list of author information is available at the end of the article
}

pared to Release 8. One such feature is a user-specific reference signal (RS) that makes it possible to suppress MU interference with a linear receiver.

With a frequency re-use factor of 1, single-cell SUand MU-MIMO network performance is highly interference limited, especially at the cell-edge. Therefore, the introduction of coordinated multipoint (CoMP) transmission/reception was already considered in Release 10. In downlink CoMP, the transmission points co-operate in scheduling and transmission in order to strengthen the desired signal and mitigate inter-cell interference. In a typical homogeneous cellular system, one site has three macro cells/sectors. Each cell has its own identification number, which is determined, for example, by the RSs that are configured for the UEs. Because of the increasing use of heterogeneous networks (HetNets), where pico cells are placed inside macro cells in order to increase network capacity, the concept of cell identity is no longer as straight forward since it is possible to assign to the picos the same cell identities as to the macro cells. Therefore, a definition of a point is needed. A point is defined as a transmission point having transmit antennas in a single geographical location [30]. Thus, one cell is formed

\section{照 Springer}

(c) 2012 Määttänen et al:; licensee Springer. This is an Open Access article distributed under the terms of the Creative Commons Attribution License (http://creativecommons.org/licenses/by/2.0), which permits unrestricted use, distribution, and reproduction in any medium, provided the original work is properly cited. 
by one or multiple points, meaning that one cell can comprise transmit antennas distributed in multiple geographical locations. In practice, the points may be base stations (evolved Node B or eNB for short) or remote radio heads (RRHs). An RRH does not include a scheduling unit but is controlled by an eNB. Figure 1 shows an example of a HetNet deployment, which has received considerable amount of attention from researchers, and which is one key scenario of interest for deploying CoMP in LTE systems.

In general, CoMP techniques have received increasing interest within the 3GPP community during Release 11 [4]. The primary focus has been on schemes called joint transmission (JT), dynamic point selection (DPS), dynamic point blanking (DPB), and coordinated scheduling/beamforming (CS/CB). In JT CoMP, two or more points transmit simultaneously to a CoMP user in a coherent or non-coherent manner. JT CoMP is depicted in Figure 2. Coherent JT means that the transmitted signals are phase aligned to achieve constructive combining of the signals at the receiver side, whereas in non-coherent JT such phase alignment is not performed. DPS refers to a scheme where the transmission point is varied according to changes in channel and interference conditions. A DPS scheme is shown in Figure 3. In CS/CB, the scheduling decisions of neighboring points are coordinated in order to reduce the interference, as in the scenario shown in Figure 4. In principle, all schemes may include point blanking/muting which means that one or more transmission points are turned off in order to decrease the interference. The overall objective of these schemes is to reduce interference and, as a result, to improve the LTE cell-edge performance. The schemes may be deployed independently or in the form of a hybrid scheme. For example, in a hybrid mode a UE may be scheduled to receive data from two points while a third point is muted, or a UE may be scheduled to receive data only from one point, but one or

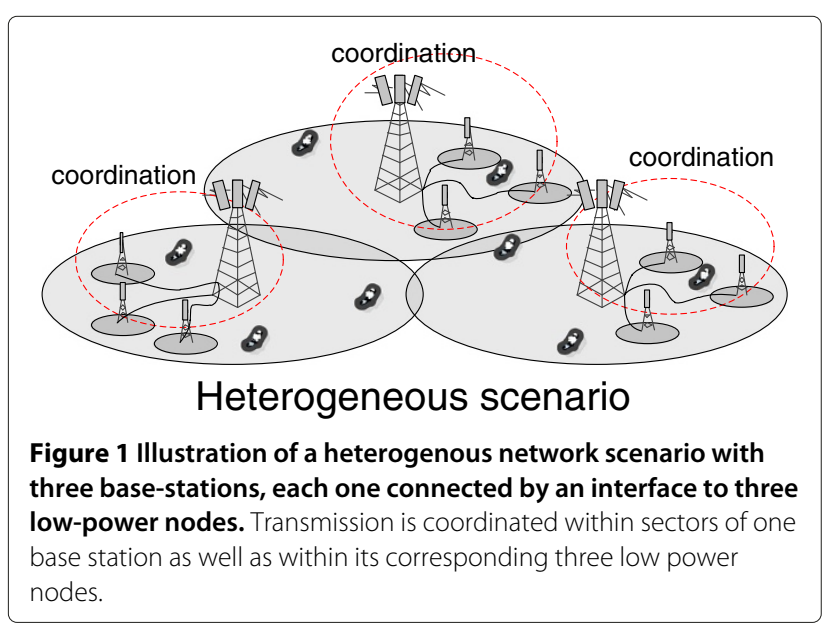

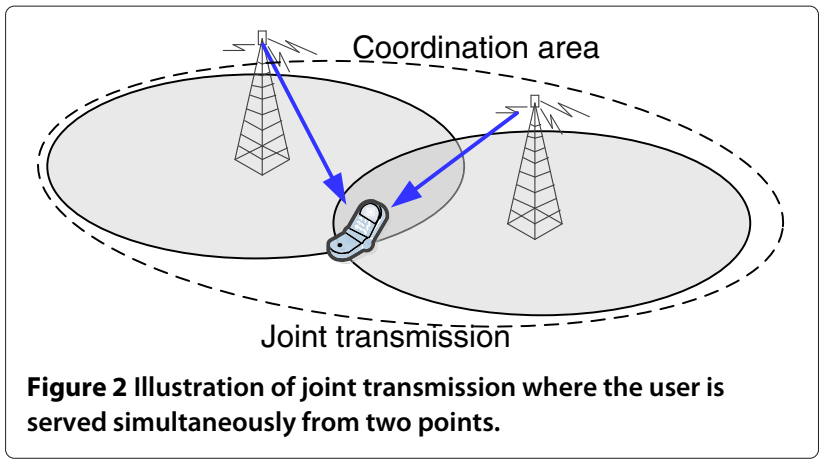

more points coordinate scheduling or are muted to reduce the interference.

There are a number of studies in the literature of CoMP in the context of LTE. A discussion paper on CS/CB, JT CoMP, and relaying can be found in [5]. In [6], JT CoMP is evaluated for increase of throughput and for energy efficiency when assuming that the channel quality indication (CQI) is derived from an accurate JT CoMP signal-to-interference-plus-noise ratio (SINR). The results show an increase of throughput at the cell edge and also $80 \%$ savings in energy efficiency per transmitted bit. In [7], a CS/CB scheme is studied for the case of full channel knowledge at the transmitter. The precoder design in this scheme exploits leakage of signal information to other cell. A similar approach has been used in [8], where JT CoMP is applied to cell-edge UEs and CS/CB to all users. In [9], interference coordination utilizing long-term channel covariance matrix information is studied. The use of long-term channelstate information (CSI) is reasonable when the cooperating points are not connected through a high-capacity and low-latency backhaul like optical fiber. Dynamic cell selection, in turn, has been studied in [10-13]. In [10], a long-term channel quality measure is used for cell selection, and in [11] the cell selection metric is a wideband short-term channel quality, equal to the averaged SINR prior to receiver processing. System-level evaluation for dynamic cell selection based on post-processing SINR values can be found for homogeneous networks

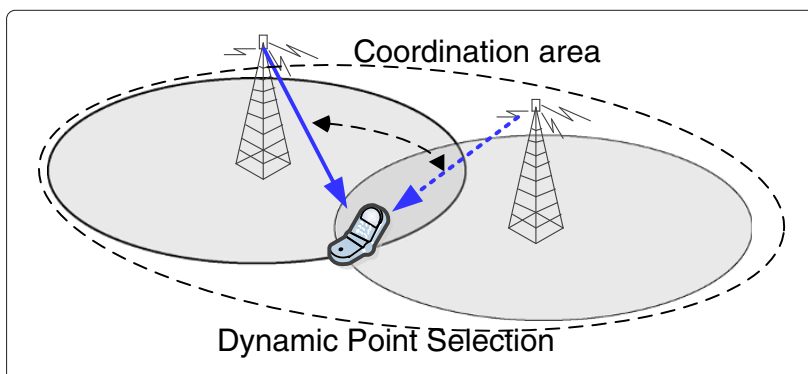

Figure 3 Illustration of dynamic point selection where the user is served by the single point with better channel conditions. 


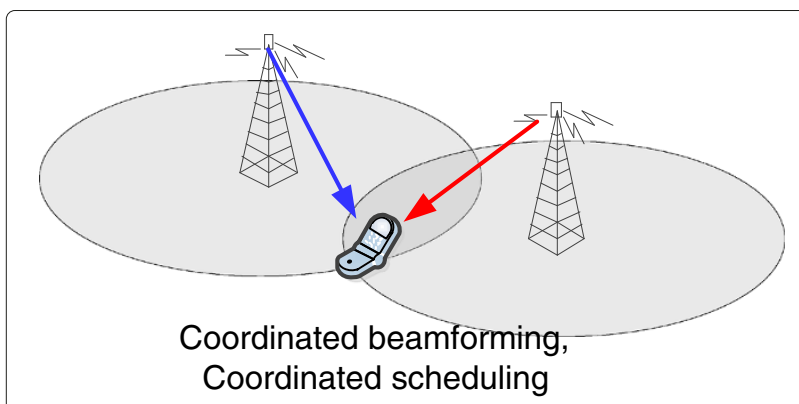

Figure 4 Illustration of coordinated beamforming and coordinated scheduling where the network coordinates beams and scheduling to avoid interference (red arrow) to a the user.

in [12] and for HetNets in [13]. The system-level results of [14] show that CoMP techniques like JT and CS/CB meet the ITU global standard for international mobile telecommunications (IMT-Advanced) performance targets. In addition, the impact of network load on CoMP network performance is studied; however, the CQI feedback is not discussed.

In [15], certain selected results from the 3GPP study item phase are shown. Some study item phase results are referred to in [16], where field test results of JT CoMP in the China 4G TDD mobile communication trial network are also presented. The results show prominent gains for JT CoMP in that TDD test network. An earlier field test for CS/CB and JT CoMP may be found in [17]. Both schemes were found beneficial and possible to implement. As future challenges to be addressed they raise the issue of backhaul assumptions, clustering and multisite scheduling, downlink feedback design and synchronization between sites. During the study item phase, assumptions varied with regard to impairments modeling and feedback. For example, the CQI feedback was assumed ideal, and even when quantized, the post-scheduling CQI was assumed to be known by the network. Thus, the effect of different CQI feedback assumptions was not studied. Currently, in the Release 11 work item stage more specific evaluations are being conducted in order to extract gains under specific feedback assumptions. The CoMP work item addresses both frequency division duplexing (FDD) and time division duplexing (TDD), hence unified solutions should be targeted, as always in the case of LTE specifications.

In this article, we look at CoMP transmission from an LTE downlink perspective, and focus in particular on the feedback signaling design and associated achievable system-level performance. Both closed-loop precoding and adaptive modulation and coding are applied to improve link performance. For closed-loop precoding, the base stations and the UEs share predefined codebooks [1]. The eNB selects the transmission weights and rates, and performs scheduling, in accordance with finite-rate user CSI feedback. The feedback consists of a CQI, a precoding matrix index (PMI) and a rank indication (RI). The CQI value represents the estimated post-processing SINR derived by the UE assuming the selected PMI. For SU single-cell transmission, the CQI estimation is straightforward, since the intercell interference is not coordinated, and therefore the level of interference estimated for CQI evaluation corresponds to the actual time of receiving the data signal. In CoMP operation, the CQI depends on the CoMP scheme and the interference hypothesis. For example, the interference level depends on CS/CB and whether or not a cooperating point is muted. Also, there exist several tradeoffs when designing the feedback for CoMP. In addition to the traditional feedback load versus performance tradeoff, one may attempt to design a unified feedback that supports all available CoMP schemes or design a scheme-specific feedback, which then requires some higher-level control or other signaling to differentiate between different CoMP modes. There exists also a tradeoff between network and UE centric operation, which means that the decision or control of the cooperation level and the specific scheme is at eNB or at UE. Typically, the network has the control but to some extent the UE is best aware of the current signal and interference conditions that it is experiencing. CQI accuracy and UE complexity also need to be taken into account. These are issues that have not so far been studied or reported systematically in the literature.

In this article, we examine the problem of feedback design and study the associated realistic system-level performance of CoMP in LTE. The higher-level starting point in this study is that different CoMP schemes require different CSI feedback. The minimum feedback needed for interference coordination is the precoder that causes the worst interference if used at the interfering point. If that precoder is known, interference may be reduced by avoiding that spatial direction. For DPS, a metric for selecting the transmission point is needed. If a UE provides feedback per point, the selection may be made in accordance with the CQI. For JT, there exist several options from per point feedback to aggregated feedback. Aggregated feedback means that the UE assumes JT transmission from $N$ points and calculates the RI, PMI, and CQI for the aggregated channel. The main contributions of this article, addressing the above fundamental challenges in practical deployment of CoMP in cellular mobile radio, are as follows: We present unified signal and system modeling to support a general hybrid CoMP scenario with varying numbers of transmission points in the JT. In an LTE compliant model, we study and propose a practical CoMP feedback design for different CoMP modes. We evaluate the tradeoff between feedback load and complexity on the one hand and the achieved performance improvements 
on the other hand. Realistic system-level performance of LTE-Advanced network is evaluated for different CoMP modes, and covers various practical deployment scenarios, including an intra-site coordination where multiple co-located sectors of an eNB are cooperating, as well as cooperation within a sector, where RRHs are operating within the coverage area of a high-power macro cell. These simulation results with realistic UE feedback indicate that CoMP is providing considerable cell-edge gains over the baseline Release 10 system. Further, when studying the CoMP schemes under biased handover conditions, it is seen that CoMP and especially DPS is a scheme that can aid in the mobility issues in real networks. This, in addition to improved cell-edge user performance, is seen as an important practical finding in this study.

The rest of the article is organized as follows. Section 2 presents the system model for LTE-Advanced and for hybrid CoMP. Section 3 describes CoMP in LTE, especially from the perspective of system and deployment scenarios, and Section 4 presents the feedback framework developed for CoMP. In Section 5, the system-level simulation results are presented. The conclusions are given in Section 6

Notations: Throughout the article, upper case bold letter $\mathbf{A}$ is used for matrices, lower case bold letter a for column vectors. $E($.) denotes expectation, $\operatorname{Re}(c)$ denotes the real part of a complex number $c, \operatorname{Tr}($.) denotes the trace of a matrix, $|\mathbf{a}|$ denotes the $\mathrm{L} 2$ norm of a vector $\mathbf{a}$ and $|a|$ denotes the absolute value of a scalar $a$.

\section{System model}

In this article, we consider the physical layer of LTEAdvanced downlink for FDD operation where the transmission scheme is orthogonal frequency division multiplexing (OFDM). In LTE-Advanced, the physical resource blocks (PRB) are defined as groups of 12 consecutive subcarriers in frequency while the subframe/transmit time interval (TTI) duration is $1 \mathrm{~ms}$ which consists of 14 OFDM symbols. Thus, the minimum timefrequency resource allocation is 12 subcarriers over 14 OFDM symbols. More details on bandwidths and subcarrier spacings, for example, can be found in $[1,18]$. As inter symbol interference may be removed using a cyclic prefix that is longer than the length of the channel impulse response, we can consider the received signal per subcarrier in frequency domain. To simplify notation, we omit the frequency and time domain indexing, and the signal model reflects subcarrier level spatial samples within one multicarrier symbol, unless otherwise stated.

\subsection{Signal model}

We consider a downlink multi-cell system with total of $M$ transmission points, where each point has $N_{\mathrm{t}}$ transmit antennas and each user has $N_{\mathrm{r}}$ receive antennas. Stating the matrix dimensions of the variables beneath the symbols, the signal $\mathbf{y}_{k}$ received by the user $k$ can be written as

$$
\underset{N_{\mathrm{r}} \times 1}{\mathbf{y}_{k}}=\underset{N_{\mathrm{r}} \times N_{\mathrm{t}}}{\mathbf{H}_{k, i}} \underset{N_{\mathrm{t}} \times r_{k}}{\mathbf{W}_{i}} \underset{r_{k} \times 1}{\mathbf{x}_{i}}+\sum_{j \neq i} \underset{N_{\mathrm{r}} \times N_{\mathrm{t}}}{\mathbf{H}_{k, j}} \underset{N_{\mathrm{t}} \times r_{j}}{\mathbf{W}_{j}}{\underset{r}{r_{j} \times 1}}_{\mathbf{x}_{j}}^{\mathbf{w}_{j}}+\underset{N_{\mathrm{r}} \times 1}{\mathbf{n}_{k},}
$$

where $\mathbf{H}_{k, i}$ is the $N_{\mathrm{r}} \times N_{\mathrm{t}}$ MIMO channel between the serving base station $i$ and user $k$, and $\mathbf{n}_{k}$ denotes the scaled noise vector whose entries are i.i.d. complex Gaussian variables with zero mean and variance $\frac{\sigma^{2}}{P}$, where $\sigma^{2}$ is the variance of additive white Gaussian noise and $P$ is the transmitted signal power. The precoding matrix $\mathbf{W}_{i}$ applied for the transmission has $r_{k}$ columns, and $r_{k}$ is the transmission rank for user $k$. The transmitted signal $\mathbf{x}_{i}$ is of length $r_{k} \times 1$. Assuming spatially uncorrelated and equal-variance transmit signal elements, we have $E\left(\mathbf{x}_{i} \mathbf{x}_{i}^{\mathrm{H}}\right)=\mathbf{I}_{r_{k}}$ and the total transmission power is controlled by precoding matrix by requiring $\operatorname{Tr}\left(\mathbf{W}_{i}^{\mathrm{H}} \mathbf{W}_{i}\right)=1$. Each element of $\mathbf{x}_{i}$, or each column of $\mathbf{W}_{i}$, corresponds to a transmission layer for user $k$. The matrices $\mathbf{H}_{k, j}$, where index $j \in\{1, \ldots, M\}, j \neq i$, are the MIMO channels between interfering transmission points and user $k$. The interfering transmission points are transmitting $r_{j}$ layers, where each signal vector $\mathbf{x}_{j}$ is precoded by the precoding matrix $\mathbf{W}_{j}$, where index $j \in\{1, \ldots, M\}, j \neq i$.

If the transmission points cooperate, the interference conditions change. For example, a UE may be scheduled to receive data from two points while the third point is muted. Alternatively, a UE may be scheduled to receive data only from one point, but one or more points coordinate scheduling or mute to reduce the interference. A general signal model for the hybrid CoMP, where $M$ is the total number of interfering points and $N \leq M$ points cooperate for user $k$, reads

$$
\begin{aligned}
\mathbf{y}_{k}= & \sum_{l=1}^{L} \mathbf{H}_{k, l} \mathbf{W}_{l} \mathbf{x}_{l}+\sum_{n=N-L+1}^{N} \alpha_{n} \mathbf{H}_{k, n} \mathbf{W}_{n} \mathbf{x}_{n} \\
& +\sum_{m=M-N+1}^{M} \mathbf{H}_{k, m} \mathbf{W}_{m} \mathbf{x}_{m}+\mathbf{n}_{k} .
\end{aligned}
$$

Here $L \leq N$ denotes the number of points that operate in JT. $N$ is the total number of points that cooperate which means that $N-L$ points cooperate by reducing interference. $M$ is the total number of points in the network. Thus, $M-N$ points are operating in an uncoordinated way with respect to the other points. The term $\alpha_{n}$ describes the level by which the interference is reduced by cooperation of the $N-L$ points, and the subscript $n$ is the point index. If $\alpha_{n}=0$ it means that point $n$ is muted and if $\alpha_{n}=1$ that point $n$ is in normal operation. 


\subsection{Single-cell operation in LTE/LTE-Advanced system}

The typical operation in LTE/LTE-Advanced is a singlecell operation which means that there is no cooperation between the eNBs. A UE selects the serving cell on the basis of received signal quality. In Release 10 LTE, different RSs are defined for channel estimation, namely CSI reference symbols (CSI-RS) and demodulation reference symbols (DM-RS). After cell selection, the eNB configures the CSI-RS and DM-RS configurations for the UE. From the CSI-RS configuration, the UE $k$ measures the MIMO channel $\mathbf{H}_{k, i}$ and calculates the CSI feedback. The DM-RS is transmitted for demodulation purposes and enables the UE to measure the effective channel $\mathbf{H}_{k, i} \mathbf{W}_{i}$.

The UE feedback consists of a wideband RI and a wideband or subband PMI and CQI. The CQI may be seen as indicative of the post-processing SINR, i.e., the SINR per stream after receiver processing. It is possible to have less independently modulated and coded data streams $N_{s}$ than there are transmitted layers $r_{k}$. In this case, one data stream is transmitted on several layers. In LTE, the maximum number of independently modulated and coded data streams $N_{s}$ is two. This means that when the number of transmission layers, or equally the transmission rank, is higher than two, a so-called layer to codeword mapping procedure is applied [1]. In this context, a codeword means a block of channel coded bits.

For the estimated MIMO channel, the UE selects a precoding matrix $\mathbf{F}_{k}^{\left(r_{k}\right)}$ of size $N_{\mathrm{t}} \times r_{k}$ from a predefined codebook and feeds back the index, PMI, as a recommendation for the serving eNB for the precoder $\mathbf{W}_{i}$. Note that with these deliberately separate notations of $\mathbf{F}_{k}$ and $\mathbf{W}_{i}$, we intend to point out that the precoder selection done by the UE is only a recommendation towards eNB. For single stream single-user transmission, the optimal choice for a precoding vector $\mathbf{f}_{k}$ for user $k$ is known to be $[19,20]$

$$
\mathbf{f}_{k}=\underset{\mathbf{f}_{p} \in \mathrm{G}_{\mathbb{C}}\left(N_{\mathrm{t}}, 1\right)}{\arg \max }\left|\mathbf{H}_{k, i} \mathbf{f}_{p}\right|^{2}
$$

where $G_{\mathbb{C}}\left(N_{\mathrm{t}}, 1\right)$ is the predefined codebook. The nested property of a codebook containing codewords for different ranks means that codewords of the codebook of higher rank include a codeword of lower rank codebook as columns. This kind of design has been introduced in order to aid rank override at the eNB. However, it depends on codeword selection metrics whether the selected codewords for higher and lower rank transmission options for the same channel realization follow the nested property. For multiple transmission layers, the optimal codeword selection criterion is a sum over the rates of the layers when the receiver processing is linear and a codeword selected with this metric does not always contain the lower rank codeword as columns [21].
In LTE-Advanced, the number of antennas at the base station may be two, four, or eight. For eight transmit antennas, the codebook has a double codeword structure $[1,22]$. One part of the codebook targets the wideband/long-term properties of the channel and the second part targets the narrowband/short-term properties. Further details of the double codebook structure are out of the scope of this article. The codebooks to support two and four downlink transmit antennas are single codebooks with separate codebooks for each transmission rank. In 4-Tx (2-Tx) case, the UE selects one precoding matrix of size $4 \times r_{k}\left(2 \times r_{k}\right)$ for rank $r_{k}$ transmission for each subband (i.e., a given number of PRBs).

The CSI feedback is derived at the UE on the basis of SU-MIMO transmission assumptions. However, MUMIMO transmission is also possible in a standard transparent manner which means that an eNB may dynamically switch between SU and MU transmission strategies based on the available single-user feedback. In general, MU transmission has a CQI mismatch problem since the postprocessing SINR depends on the precoding matrix used for multiplexing the users which depends, in turn, on the eNb scheduling decision [23-25]. Therefore, MU performance is greatly affected by the outer loop link adaptation (OLLA) algorithm [26] which tunes the link adaptation during the CQI reporting period based on ACK/NACK received from the UE.

Similarly, an MU CoMP can be considered in a standard transparent way. For DPS and CS/CB, the MU scenario has similar issues as for single-cell transmission. For JT CoMP, there is an additional power allocation problem if the zero forcing beamforming is used [27]. In this article, we consider SU single-cell MIMO operation as the baseline against which the SU CoMP methods are compared in terms of network performance.

\section{CoMP in LTE-Advanced}

Users in CoMP mode receive data from one or multiple points in the coordination area, hence prior to receiving the data, they need to report the CSI feedback for these coordinated points. A CoMP measurement set is formed by the $N$ cells/points for which the UE is measuring the CSI. For Release 11, the maximum CoMP measurement set size is $N=3$. The point from which the UE would receive transmission in single point mode is defined as the serving/fallback point.

In addition to the information exchange between the users and the transmission points, the cooperation requires information exchange between the cooperating points or a common scheduling entity that controls the set of cooperating points. The information that needs to be shared includes UE CSI feedback, scheduling decisions, and possible user data. All delays in the information exchange affect the CoMP operation and especially 
exchanging the user data between the points may require some extra capacity from the backhaul link. In addition, the requirement for JT and DPS is that the user data is available and synchronized in the transmission points participating in JT or DPS for a particular UE. Especially, the synchronization of the user data requires fairly ideal backhaul both in capacity and delay. Iterative CS/CB schemes are also prone to extra delays of the backhaul. The CoMP operation specified in Release 11 assumes ideal fiber connection between the points that may cooperate. From the backhaul perspective this enables JT and DPS as well as iterative CS/CB CoMP methods. The effects of a nonideal backhaul and the X2 interface are to be evaluated in Release 12 . The X2 interface is a protocol stack defined in the LTE standard for connecting eNBs [28]. The purpose of the $\mathrm{X} 2$ interface is to enable information exchange between different vendors' eNBs. The schemes that can be envisioned operating over non-ideal backhaul and requiring information exchange over X2 are for example simple non-iterative CS/CB schemes, where eNBs simply avoid scheduling UEs that would likely cause strong interference to each other. These schemes need PMI feedback in the form of short-term feedback, or long-term interference covariance matrix CSI. The typical X2 backhaul average latency is $10 \mathrm{~ms}$; however, the latency may also be around $20 \mathrm{~ms}$ [29]. For comparison, the subframe length is $1 \mathrm{~ms}$ and CSI feedback may be triggered with $5 \mathrm{~ms}$ periodicity. Thus, the scheduling decisions and consequently the interference conditions may vary rapidly even if the channel was more stable, e.g., for low mobility users. For these reasons, the short-term feedback might not be convenient due to the aging problem of the CSI report if exchanged through X2 backhaul.

\subsection{CoMP network scenarios}

The agreed CoMP work item targets specification of intraand inter-cell DL CoMP schemes operating in homogeneous and HetNet deployments [30]. Four main scenarios have been studied so far

- intra-site scenario where multiple co-located sectors of the same eNB site are cooperating (Scenario 1), illustrated in Figure 5,

- inter-site scenario with high-power RRHs where multiple non-co-located points having the same transmit power are cooperating (Scenario 2), illustrated in Figure 6,

- low-power RRHs within the coverage of the high-power macro cell, each operating its own cell ID (Scenario 3), illustrated in Figure 1, and

- low-power RRHs within the coverage of the high-power macro cell, each operating with the same cell ID (Scenario 4). In [31], Scenario 4 is discussed in

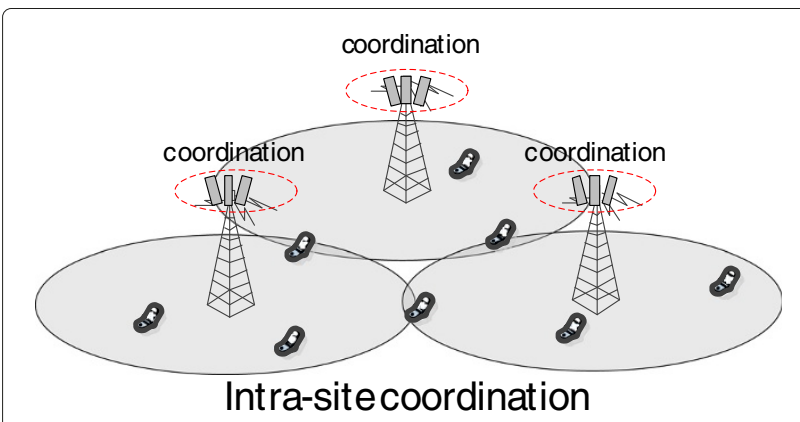

Figure 5 Illustration of intrasite coordination where transmission is coordinated within sectors of one base station.

detail and results from the study item phase are presented.

During Release 11 time frame, only cooperation between transmission points controlled by one scheduling unit is possible due to the fiber connection assumption. For the homogeneous scenarios, UEs are dropped uniformly in the macro sector area. For the HetNet scenarios, two different UE dropping methods are defined [18]:

- Configuration 1: 25 UEs uniformly dropped in the macro sector geographical area.

- Configuration 4b: clustered UE dropping with total of 30 UEs, $1 / 3$ of the UEs dropped uniformly in the macro sector geographical area and $2 / 3$ of the UEs dropped inside a $40-\mathrm{m}$ radius of pico points.

\subsection{RSs for CoMP in LTE}

In Release 11, it has been agreed that the UE may receive multiple CSI-RS configurations corresponding to the points in the measurement set. One CSI-RS configuration corresponds typically to transmission from one point, but it is possible to configure two transmission points under one CSI-RS configuration transparently to a UE. For example, there can be two 2Tx transmission points

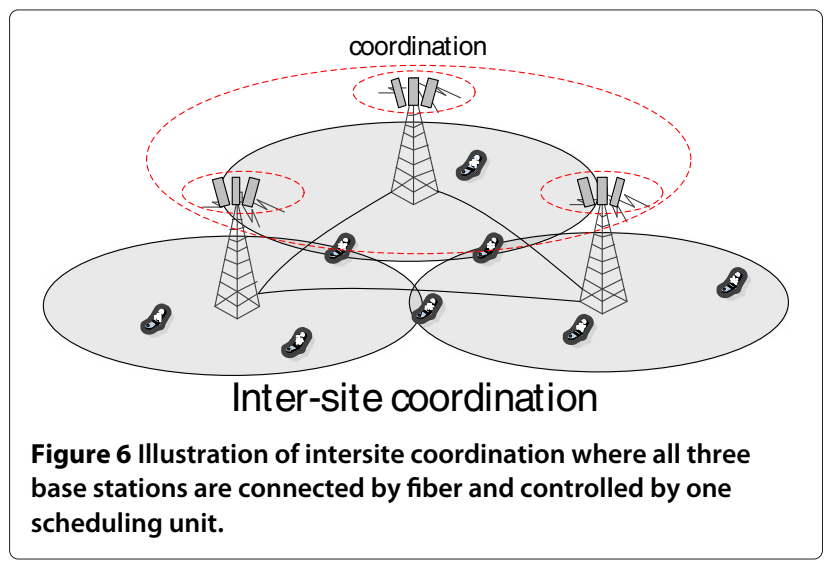


that can be configured to a UE as two separate transmission points or as one virtual $4 \mathrm{Tx}$ transmission point. In addition, a term CSI-RS resource is defined as a CSIRS configuration and an interference assumption, which provides a CQI assumption.

For selecting the points forming the CoMP measurement set, an eNB can monitor the uplink signal received powers, for example through sounding RSs. As multiple transmission points are connected to a centralized CoMP scheduler that receives the sounding RSs, a classification can be made of the link qualities for the points involved in a CoMP cluster. After this, the best two or three points that are reliable for CoMP transmission are selected. The reliability of a point is defined such that the link power is within an $X \mathrm{~dB}$ power window (usually of 5-6 dB) from the serving point link power. Alternatively, the UEs may compute and report the received power value of the CSI-RS, that is receiver power for the CSIRS transmission from points in CoMP cluster. The eNB then selects the best points which are the most suitable for CoMP transmission.

\section{CSI feedback in CoMP}

After measuring the channels of the cooperating points, UE derives the RI, PMI, and CQI feedback. The feedback can be derived per CSI-RS configuration, that is per point. In addition, it is possible to configure the CSI-RS over multiple points, a UE being configured to calculate feedback over geographically separated antennas in a standard transparent manner. This feature is not evaluated in this article and is left for future work.

Here, we select and feed back per point PMIs, because in this way existing per point single-cell codebooks can be reused. In addition, we select the per point PMIs independently. Joint per point PMI selection for JT transmission has been proposed in [32]. While joint per point PMI selection improves the performance of JT transmission compared to independent per point PMI selection, such a joint selection increases the selection complexity and moreover is suboptimal for DPS and fallback transmission. In [33], Stiefel-Grassmannian per-point codebooks have been proposed together with Stiefel distance selection metric used for the second/weaker transmission point. The proposed Stiefel distance selection metric balances between maximizing the received power and maximizing the coherency of the transmission. The performance of JT transmission is improved; however, the selected codeword for the second/weaker point is no longer optimal for single point transmission. With the per point independently selected PMIs, being a unified feedback, we study the need for additional inter-point PMI feedback for JT transmission and different CQI feedback options for JT and DPS CoMP. In CoMP operation, the
CQI depends on the CoMP scheme and the interference hypothesis. That is, the CQI depends on $L, N$, and the interference assumption in Equation (2). The size of the measurement set, $N$, is known by the UE as the network configures the CSI-RS resources for it.

\subsection{CQI feedback options}

Reducing the interference is beneficial for the selected transmission rate because improved signal conditions increase the reliability of the link. However, from the link adaptation point of view, especially if there is a clear improvement in the interference conditions, as for example due to muted points, full advantage can only be gained if the CQI feedback reflects the improved link quality. Therefore, precise CQI information capturing the interference conditions accurately is important from the performance point of view even though OLLA can, to some extend, compensate CQI inaccuracies.

From a feedback design point of view, the $N=2$ case already results in several CQI options as shown in Table 1, where $S$ and $I$ denote the respective signal and interference powers. Considering that the CSI-RS is configured per point and the UE selects one PMI per point, then it is possible to derive several different CQIs to support different CoMP schemes simultaneously. The UE may derive an aggregated CQI for the JT transmission and multiple CQIs per point with different interference assumptions, thus making use of different $\alpha$ values. If $N=3$, the CQI options are shown in Table 2 where there are four different CQI options for the JT transmission, i.e., JT from all three points and JT from two out of three points, all with possible different interference assumption from the third point. In addition, there are per point CQIs with different interference assumption combinations from the two cooperation points. Note that if $\alpha<1$ for the CQI for the serving point, then an additional fallback CQI is needed for the serving point to secure the baseline single-cell transmission.

It is clear that full CQI feedback supporting all transmission options is not feasible as the number of CQIs may grow enormously. Note that the CQIs discussed above are per independently modulated and coded data stream, thus rank two transmission assumption for one scheme would

\begin{tabular}{|c|c|c|}
\hline & Point 1 & Point 2 \\
\hline DPS & $S$ & $\alpha \times 1$ \\
\hline DPS & $\alpha \times 1$ & $S$ \\
\hline JT & $S$ & $S$ \\
\hline
\end{tabular}


Table 2 CQI options for three points, where $\alpha$ expresses interference assumption

\begin{tabular}{llll}
\hline & Point 1 & Point 2 & Point 3 \\
\hline DPS & $S$ & $\alpha \times 1$ & $\alpha \times 1$ \\
DPS & $\alpha \times 1$ & $S$ & $\alpha \times 1$ \\
DPS & $\alpha \times 1$ & $\alpha \times 1$ & $S$ \\
JT & $S$ & $S$ & $\alpha \times 1$ \\
JT & $\alpha \times 1$ & $S$ & $S$ \\
JT & $S$ & $\alpha \times 1$ & $S$ \\
JT & $S$ & $S$ & $S$ \\
\hline
\end{tabular}

mean two CQIs for that scheme instead of one. In addition, CQI may be per subband. Hence, the rank utilization, feedback frequency granularity, and the number of points for which CSI feedback is computed are all factorizing the overall feedback overhead that needs to be sent from the receiver to the transmitter. In the following section, we conduct further analysis of these topics.

\subsection{Tradeoffs in CoMP feedback design}

The traditional tradeoff between feedback load versus performance relates to the tradeoff between network centric and UE centric CoMP. The UE centric CoMP refers to the operation where the UE selects the coordination set and the preferable CoMP scheme based on channel and interference measurements and sends the corresponding feedback. The advantages are that because the UE has the instantaneous knowledge on the downlink channel and interference conditions, it may deduce the best CoMP feedback for these conditions. Thus, feedback savings are possible in principle because, for example, a UE could send feedback only when the channel conditions are good and only for specific CoMP schemes. From the network perspective, the richer the feedback the scheduler entity has, the better the expected network performance is. If the network may receive information from every active UE and it has, for example, information about the number of served UEs and achieved transmissions rates, it can more efficiently evaluate which CoMP schemes should be applied. This could be beneficial in enabling a flexible balance between transmission methods to the users. Thus, receiving feedback for multiple CoMP transmission hypothesis from one UE would be beneficial. When considering network centric CoMP, which is the commonly supported method, higher layer signaling should be considered as well. This means that the CoMP operation can be designed either transparent to the UE meaning that the UE always feeds back certain CQIs based on CSI-RS resources configured for it, or the UE may be configured by higher protocol layers to calculate a scheme-specific feedback.

\subsection{CoMP scheduling}

In 3GPP, the signaling and feedback between the network and the users are specified but the packet scheduler is an eNB implementation-specific feature. The performance of an LTE/LTE-Advanced system largely depends on the packet scheduling algorithm applied at the network side. In the system-level evaluations of this article, a proportionally fair (PF) packet scheduler with properly tuned scheduling parameters is used with the aim of maximizing the baseline Release 10 performance. A single point PF scheduler is analyzed and described in detail in [34]. If CoMP is enabled, the same baseline PF scheduling with the same parametrization is used in the first stage to find the single-cell candidates to be scheduled, while in the second stage a CoMP-specific scheduling is performed.

All the JT CoMP reporting UEs are sorted according to their PF-metrics derived from CoMP feedback. The highest JT CoMP PF-metric in a given subband is compared against the sum of single-cell users', also called the victim users', PF-metrics. If the JT CoMP PF metric is higher than the sum of victim UE's metrics, CoMP UE is scheduled and victim UEs allocations are altered accordingly. This scheduling algorithm is applied for each subband. DPS CoMP allocates resources to UE from the point in which UE reported the highest instantaneous wideband CQI. OLLA and UEs scheduling history are assumed to be shared between the points with no delay. In addition, the network is assumed to be fully synchronized.

\subsection{Feedback to support DPS CoMP}

The feedback to support DPS CoMP is per point feedback including RI, PMI, and CQI. PMIs are derived normally as for single-cell transmission and CQI is derived from the SINR value. SINR for user $k$ from point $i$ with single stream transmission assumption may be written as

$$
\begin{aligned}
\operatorname{SINR}_{k, i}^{\mathrm{DPS}}\left(\alpha_{n}\right)= & \frac{\left|\mathbf{g}_{k}^{\mathrm{H}} \mathbf{H}_{k, i} \mathbf{w}_{i}\right|^{2}}{\left|\mathbf{g}_{k}^{\mathrm{H}} \sum_{n=N-L+1}^{N} \alpha_{n} \mathbf{H}_{k, n} \mathbf{W}_{n}\right|^{2}}, \\
& +\left|\mathbf{g}_{k}^{\mathrm{H}} \sum_{m=M-N+1}^{M} \mathbf{H}_{k, m} \mathbf{W}_{m} \mathbf{x}_{m}\right|^{2}+\sigma^{2}
\end{aligned}
$$

where $\mathbf{g}_{k}$ is the normalized receiver combiner for user $k$ and $\sigma^{2}$ is the noise variance. The CQI feedback options for DPS are relatively simple since DPS refers to single point transmission with possible muting assumptions from the cooperating points. For CoMP with two cooperating points there are two CQI options for both points. The cooperating point may be muted or transmitting normally. We refer to these options as $\mathrm{CQI}_{k, i}^{\mathrm{DPS}}$ when one point is not muted and $\mathrm{CQI}_{k, i}^{\mathrm{DPB}}$ when the other point is muted. The DPS feedback can be network centric or UE centric. In the network centric option, the UE feeds back per point feedback to all points and in the UE centric option only to 
Table 3 Link-level simulation assumptions

\begin{tabular}{ll}
\hline Parameter & Assumptions used for evaluation \\
\hline Scenario & 21 sector hex eNB grid + 4 RRHs per sector \\
Channel for eNB & ITU UMa LOS/NLOS [36] \\
Channel for RRH & ITU UMi LOS/NLOS [36] \\
UE speed & $1 \mathrm{~km} / \mathrm{h}$ \\
Tx point \# of antennas & $2, \mathrm{X}-\mathrm{pol} \pm 45 \mathrm{deg}$ \\
UE \# of antennas & $2, \mathrm{X}-$ pol, 0.5 $\lambda, 0 / 90$ deg \\
Measurement set & 2 strongest, 6-dB threshold \\
Scheduler & Round Robin, only CoMP users \\
HARQ & 2 retransmissions \\
CSI Estimation & Ideal \\
Receiver & MMSE \\
Codebook & $3 G P P 2$ Tx [1] \\
Feedback & $6 P R B$ granularity, no delay, ideal CQI \\
OLLA step-up/down & No delay, $\frac{1}{9} / 1$ dB \\
\hline
\end{tabular}

the strongest point. Special care needs to be taken when thinking about fallback/single-cell performance, because the single-cell operation is performed also in CoMP eligible cells. A fallback point means that the serving point and the corresponding feedback should be Release 10 specific. Release 10-specific CQI refers to the case where no muting or other cooperation form is applied, that is $\alpha_{n}=$ $1, \forall n$. The importance of always feeding back the fallback $\mathrm{CQI}$ is evaluated and illustrated in the results section.

\subsection{Feedback to support JT CoMP}

For JT CoMP, the comparison between the aggregated feedback and per point feedback is highly relevant. JT transmission is possible with per point PMI and CQI feedback. In this case, the transmitter would combine the PMIs and CQIs for the JT transmission. It is expected that inter-point feedback and aggregated CQI would improve performance for JT CoMP. In the next sections, we present various precoding and CQI feedback options for JT CoMP.

\subsubsection{PMI feedback and inter-point combiner for JT}

The simplest form of the PMI feedback is per CSI-RS resource feedback. From a transmission perspective, each point is independently transmitting the same data to the user, hence coherent transmission is not possible without additional feedback. The additional feedback required for coherent transmission is an inter-point combiner describing the amplitude and phase of that transmission. The inter-point combiner for point $n$ for single stream transmissions can be written as

$$
c_{n}=a_{n} e^{j \theta_{n}},
$$

where $\theta_{n}$ is the inter-point phase combiner and $a_{n}$ is the inter-point amplitude. The combiner phase is always a relative quantity, thus without loss of generality we may select $\theta_{1}=0$ always. For multi-stream transmission the combiner can be defined per transmission layer, or in the most general form, as a matrix of dimension $r_{k} \times r_{k}$, where the off diagonal elements characterize the inter-layer effects. The transmission equation (2) for

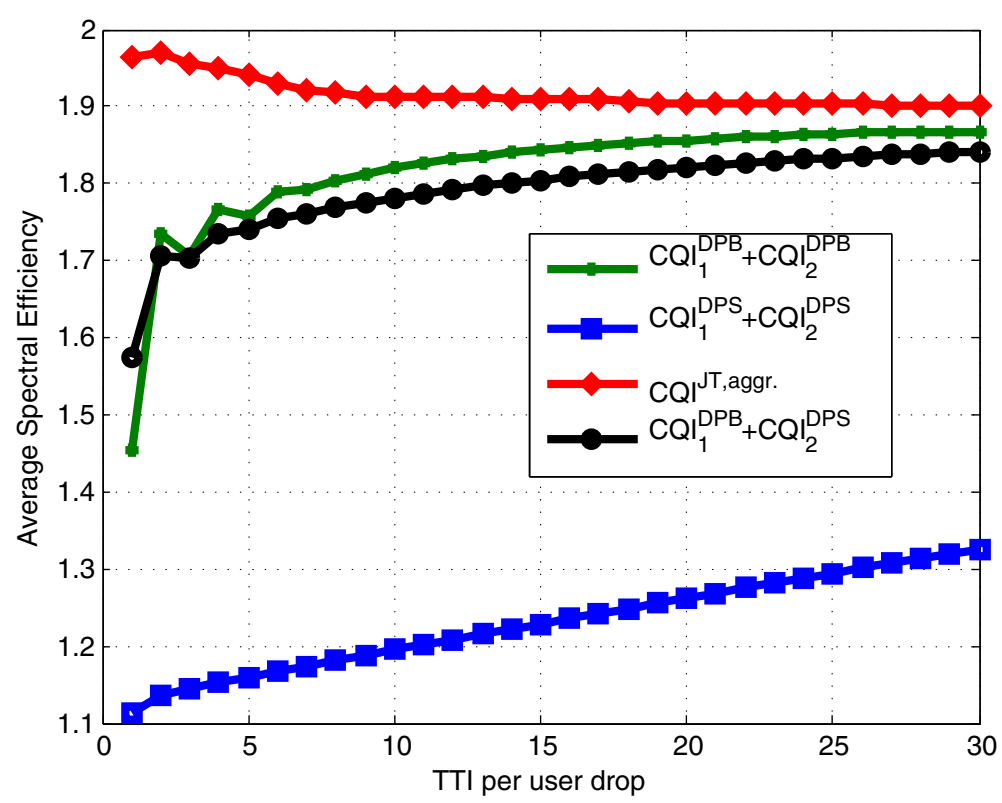

Figure 7 Extended link performance of non-coherent JT with several different CQI feedback hypotheses as a function of a scheduled link duration. OLLA mechanism corrects CQI mismatch at the transmitter. 


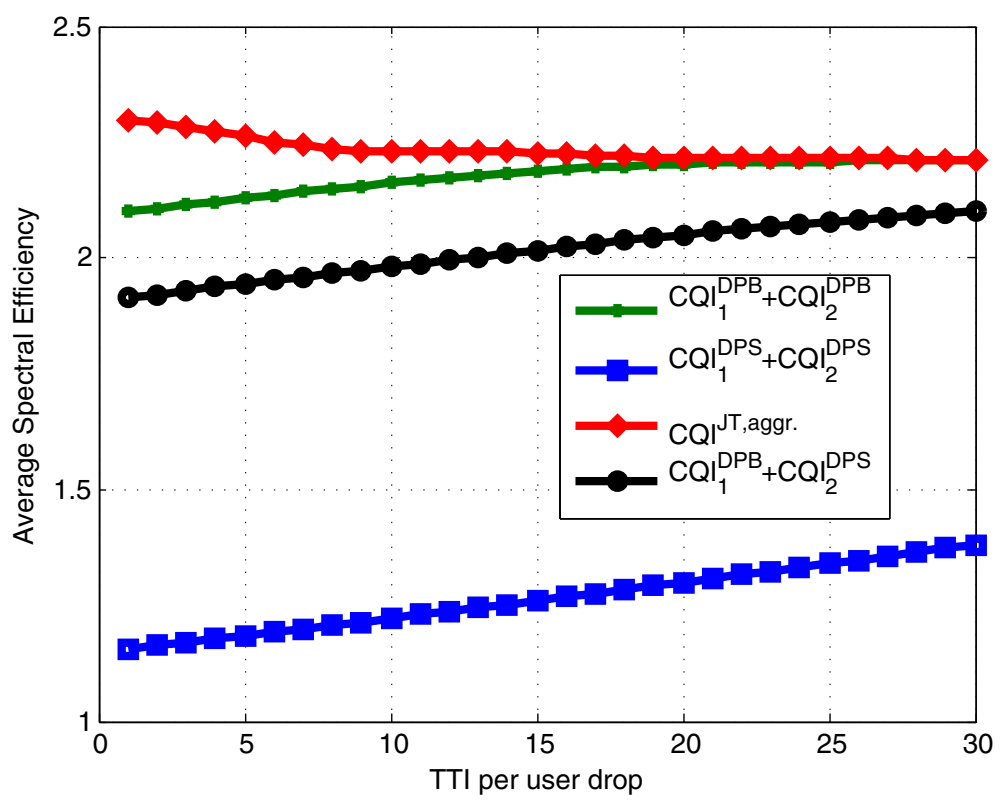

Figure 8 Extended link performance of JT transmission with QPSK combiner and different CQI feedback hypotheses as a function of a scheduled link duration. OLLA mechanism corrects CQI mismatch at the transmitter.

single stream transmission, where all cooperating points perform JT, $N=L$, can be written as

$$
y_{k}=\sum_{n=1}^{N} c_{n} \mathbf{h}_{k, n}^{\mathrm{eff}} x_{k}+\sum_{m=M-N+1}^{M} \mathbf{h}_{k, m}^{\mathrm{eff}} x_{m}+\mathbf{n}_{k},
$$

where $\mathbf{h}_{k, n}^{\text {eff }}=\mathbf{H}_{k, n} \mathbf{w}_{n}$ is the precoded channel between the $k$ th user and $n$th transmission point. For the two transmission points case, i.e., $N=2$, optimal amplitude combiners $a_{n}$ can be selected as in [35]. In practice, however, the power pooling between transmission points is not possible, because total transmission power at the transmission point cannot be exceeded due to system specifications and regulatory issues. If the resources at both transmission points have been scheduled to a single user, it is from a user perspective always worth

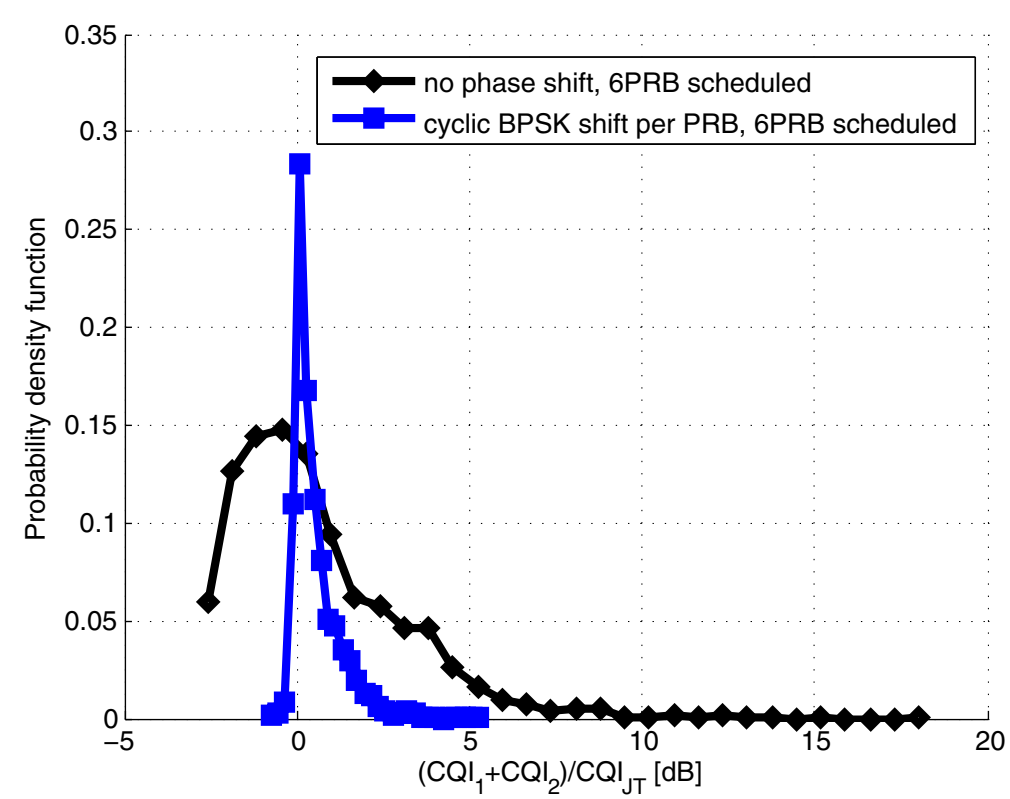

Figure 9 Cumulative density function of CQI mismatch with and without BPSK cyclical phase shift. 


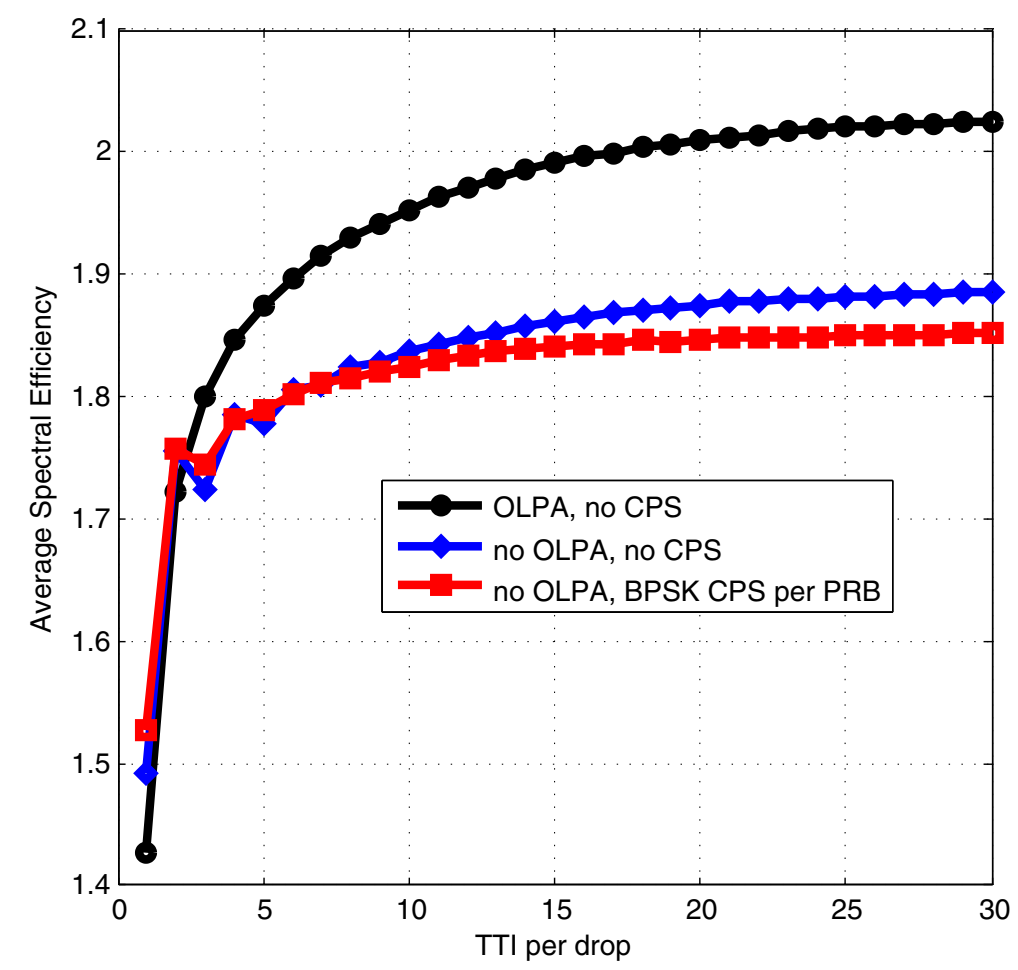

Figure 10 Extended link performance of non-coherent JT transmission with/without OLPA correction.

transmitting from both transmission points with full power rather than muting the weaker transmission point completely. Therefore, in the rest of the article, we will set $a_{n}=1$. For $N=2$, which is the primary case in this article, we employ optimal combiner phase $\theta_{2}$ quantized uniformly with $B$ bits. The optimal combiner phase $\theta_{2}$ maximizes the norm of the sum of two effective channels $\left|\mathbf{h}_{k, 1}^{\text {eff }}+e^{j \dot{\theta}_{2}} \mathbf{h}_{k, 2}^{\text {eff }}\right|$ only when

$$
\operatorname{Re}\left\{\mathbf{h}_{k, 1}^{\text {efff }} \mathbf{h}_{k, 2}^{\text {efff }} e^{j \theta_{2}}\right\}=\left|\mathbf{h}_{k, 1}^{\text {eff }} \mathbf{h}_{k, 2}^{\text {eff }}\right| .
$$

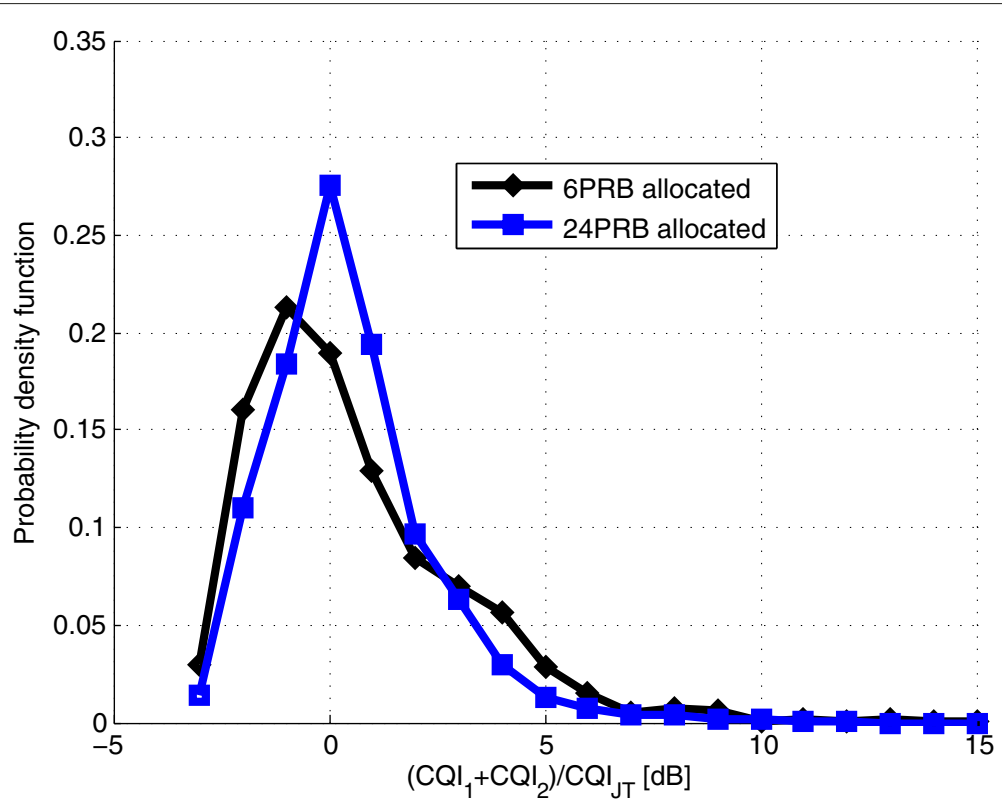

Figure 11 Cumulative density function of CQI mismatch with 6/24PRB scheduled bandwidth. 
Table 4 Simulation assumptions for system-level evaluations

\begin{tabular}{|c|c|}
\hline Parameter & Value \\
\hline \multirow[t]{2}{*}{ Cellular layout } & $\begin{array}{l}\text { Hexagonal grid, } 19 \text { sites, } 3 \text { sectors per } \\
\text { site, }\end{array}$ \\
\hline & $\begin{array}{l}\text { Center site simulated, } 500 \mathrm{~m} \text { inter site } \\
\text { distance }\end{array}$ \\
\hline Traffic model & Full buffer \\
\hline \multirow[t]{2}{*}{ Deployment scenarios } & $\begin{array}{l}\text { CoMP Scenario } 3 \text { according to } 3 \mathrm{GPP} \\
36.819 \text { v. 11.1.0 }\end{array}$ \\
\hline & $\begin{array}{l}\text { Coordinated TX-points } 3 \text { macros }+12 \\
\text { picos }\end{array}$ \\
\hline Carrier frequency & $2.00 \mathrm{GHz}$ \\
\hline Antenna configuration & 2 Tx cross polarized (XPOL), 2 Rx XPOL \\
\hline \multirow[t]{3}{*}{ Number of UEs } & $\begin{array}{l}\text { Configuration 1: } 25 \text { UEs / macro geo- } \\
\text { graphical area. }\end{array}$ \\
\hline & $\begin{array}{l}\text { Configuration 4b: } 30 \text { UEs / macro geo- } \\
\text { graphical area. }\end{array}$ \\
\hline & $\begin{array}{l}\text { UE dropping according to 3GPP } 36.814 \\
\text { v. 9.0.0. }\end{array}$ \\
\hline \multirow[t]{2}{*}{ Transmission schemes } & SU-MIMO with JT \\
\hline & SU-MIMO with DPS \\
\hline UE receiver & 3GPP option 1 \\
\hline $\begin{array}{l}\text { Channel estimation for } \\
\text { feedback }\end{array}$ & Realistic CSI-RS based \\
\hline
\end{tabular}

\begin{tabular}{|c|c|}
\hline $\begin{array}{l}\text { Channel estimation for } \\
\text { demodulation }\end{array}$ & Realistic through AVI tables \\
\hline \multirow[t]{4}{*}{ UE Feedback } & Rank indicator, max rank 2. \\
\hline & $\begin{array}{l}\text { CoMP transmission rank same as serving } \\
\text { TX-point rank }\end{array}$ \\
\hline & $\begin{array}{l}\text { Mode 3-1: Subband (6 PRB) CQI, Wide- } \\
\text { band PMI }\end{array}$ \\
\hline & $\begin{array}{l}6 \mathrm{~ms} \text { delay and } 10 \mathrm{~ms} \text { interval for } \mathrm{CQ} \text { and } \\
\text { PMI }\end{array}$ \\
\hline CoMP reporting threshold & $\begin{array}{l}\text { TX-points having RS received power } \\
\text { inside } 6 \mathrm{~dB} \text { window }\end{array}$ \\
\hline $\begin{array}{l}\text { Max. CoMP measurement } \\
\text { set size }\end{array}$ & 2 TX-points \\
\hline $\begin{array}{l}\text { Reference symbol } \\
\text { overhead }\end{array}$ & DM-RS: 12 RE PRB \\
\hline
\end{tabular}

DM-RS ports CSI-RS: 2 RE/PRB per $10 \mathrm{~ms}$

CRS: 2 CRS Rel8 legacy overhead

\begin{tabular}{ll}
\hline Control channel & $\begin{array}{l}\text { Only overhead modelled: 3 OFDM } \\
\text { symbols }\end{array}$ \\
\hline Scheduler algorithm & PF \\
\hline Interference modelling & $\begin{array}{l}\text { Random rank and PMI in interfering Tx- } \\
\text { points }\end{array}$ \\
\hline OLLA & Enabled, BLER target 10\% \\
\hline HARQ & Max 4 retransmission, chase combining \\
\hline
\end{tabular}

While aggregated PMI across all received CSI-RS resources may offer better feedback compression/ performance compared to per CSI-RS resource feedback, it has several drawbacks. First, codebooks for various combinations of transmit points with different antenna configurations and types needs to be designed. Second, the aggregated PMI selected with the JT hypothesis is not optimal for DPS and CS/CB schemes. Unlike the aggregated PMI, the per-point PMI feedback may be improved by the additional combiner (inter-CSI-RS resource) feedback. Although the separately coded inter-point feedback with combiner may require additional feedback compared to the aggregated PMI, it does not require new codebooks to be designed and such a feedback is optimal for DPS $\mathrm{CS} / \mathrm{CB}$ transmission schemes as well.

\subsubsection{CQI feedback for JT}

The JT CQI used for JT may be estimated from per-cell CQIs or an additional aggregated JT CQI (CQI $\left.{ }^{\mathrm{T} \text {,aggr. }}\right)$ can be fed back. The aggregated SINR for JT for user $k$ can be expressed as

$$
\operatorname{SINR}_{k}^{\text {JT,aggr. }}=\frac{\left|\mathbf{g}_{k}^{\mathrm{H}} \sum_{n=1}^{N} c_{n} \mathbf{h}_{k, n}^{\text {eff }}\right|^{2}}{\left|\mathbf{g}_{k}^{\mathrm{H}} \sum_{m=M-N+1}^{M} \mathbf{h}_{k, m}^{\mathrm{eff}}\right|^{2}+\sigma^{2}} .
$$

From Equation (8), we note that $\mathrm{SINR}_{k}^{\mathrm{JT}, \mathrm{aggr} .}$ is a function of the channel gains. The channel gains or the channels are not available at the transmitter as such but it is convenient to assume such availability in this discussion. For two transmission points and single stream transmission, the channel gain $G_{k}^{\mathrm{JT}}$ for the user $k$ can be written as

$$
\begin{aligned}
G_{k}^{\mathrm{JT}}= & \left|\mathbf{h}_{k, 1}^{\mathrm{eff}}+\mathbf{h}_{k, 2}^{\text {eff }}\right|^{2}=\underset{\text { Channel gain 1 }}{\left|\mathbf{h}_{k, 1}^{\mathrm{eff}}\right|^{2}}+\underset{\text { Channel gain 2 }}{\left|\mathbf{h}_{k, 2}^{\mathrm{eff}}\right|^{2}} \\
& +\underset{\text { constructive/destructive addition . }}{2 \operatorname{Re}\left\{\mathbf{h}_{k, 1}^{\mathrm{eff}} H\right.} c_{\left.c_{2} \mathbf{h}_{\text {eff }}^{\text {eff }}\right\}}
\end{aligned}
$$

Plugging the first two channel gains into the nominator of the SINR equation (4) for DPB transmission, we may rewrite the $\mathrm{SINR}_{k}^{J \mathrm{~T}}$ as

$$
\operatorname{SINR}_{k}^{\text {JT,aggr. }}=\operatorname{SINR}_{k, 1}^{\mathrm{DPB}}+\mathrm{SINR}_{k, 2}^{\mathrm{DPB}}+\mathrm{SINR}_{\Delta},
$$

where $\mathrm{SINR}_{\Delta}$ is a CQI mismatch which corresponds to the constructive/ destructive addition of the channels from the two points. In other words, if the third term of Equation (9) is negative, the channel addition is destructive and $\mathrm{SINR}_{\Delta}$ is negative. When the term is positive, the addition is constructive and $\mathrm{SINR}_{\Delta}$ is positive. The constructiveness/destructiveness depends on the phase between the effective channel vectors and makes the $\mathrm{SINR}_{\Delta}$ positive/negative with $50 \%$ probability assuming no inter-point feedback information is used.

In Equation (9), per-cell CQIs with muting hypothesis are used. In order to investigate the impact of CQI 
Table 5 Simulated CQI options

\begin{tabular}{|c|c|c|c|}
\hline CQI feedback & $\begin{array}{l}\text { Primary point } \\
C Q I_{1}\end{array}$ & $\begin{array}{l}\text { Cooperating point } \\
\mathrm{CQI}_{2}\end{array}$ & Remarks \\
\hline $\mathrm{CQ}_{k}^{\mathrm{J}, \text { aggr. }}, \mathrm{CQI}_{k, 1}^{\mathrm{Rel}} 10$ & $\frac{S_{1}}{l_{\text {out }}+N+S_{2}}, \frac{S_{\int_{1} \text {, aggr. }}}{l_{\text {out }}+N}$ & - & Optimal for JT \\
\hline $\mathrm{CQI}_{k, 1}^{\mathrm{DPS}}, \mathrm{CQI}_{k, 2}^{\mathrm{DPS}}$ & $\frac{S_{1}}{l_{\text {out }}+N+S_{2}}$ & $\frac{S_{2}}{l_{\text {out }}+N+S_{1}}$ & Rel 10 CQls \\
\hline $\mathrm{CQ}_{k, 1}^{\mathrm{DPB}}, \mathrm{CQ}_{k, 2}^{\mathrm{DPB}}$ & $\frac{S_{1}}{l_{\text {out }}+N}$ & $\frac{S_{2}}{l_{\text {out }}+N}$ & No correct fallback \\
\hline $\mathrm{CQ}_{k, 1}^{\mathrm{DPB}}, \mathrm{CQ}_{k, 2}^{\mathrm{DPS}}$ & $\frac{S_{1}}{T_{\text {out }}+N+S_{2}}$ & $\frac{S_{2}}{l_{\text {out }}+N}$ & Correct fallback \\
\hline $\mathrm{CQI}_{k, 1}^{\mathrm{DPB}}, \mathrm{CQ}{ }_{k, 1}^{\mathrm{Rel}} 10,\left.\mathrm{CQ}\right|_{k, 2} ^{\mathrm{DPB}}$ & $\frac{S_{1}}{\text { lout }+N}, \frac{S_{1}}{\text { lout }_{\text {on }}+N+S_{2}}$ & $\frac{S_{2}}{l_{\text {out }}+N+S_{2}}$ & Feedback load increased \\
\hline
\end{tabular}

mismatch on the link performance, extended link simulations have been carried out under various CQI feedback hypotheses. The main simulation assumptions are summarized in Table 3. The simulation procedure is as follows: Four RRHs are dropped into every sector of the hexagonal macro network. The users are dropped non-uniformly (Configuration 4b) into the middle site until a user satisfying the CoMP threshold is found. Network generation and user dropping are according to Scenario 3/4 in [18]. The found CoMP user is scheduled in JT CoMP mode and its feedback is computed. Finally, a pre-defined number of TTIs is simulated while OLLA is employed.

Figure 7 shows the performance of the estimated CQI for several settings of muting hypothesis. In the case that the $\mathrm{CQI}^{D P B}$ are fed back, performance suffers only minor degradation. A similar investigation has been run with a QPSK combiner. Figure 8 shows that with the QPSK combiner, the CQI mismatch can be kept even smaller and the performance of $\mathrm{CQI} \mathrm{I}^{\mathrm{T} \text {,aggr. }}$ can already be reached within 20 iterations of OLLA algorithm. The CQI mismatch with $\mathrm{CQI}^{\mathrm{DPB}}$ feedback can be minimized by the following approaches

1. Adapting the phase combiner (BPSK) with outer-loop-phase-adaptation (OLPA);

2. Cyclical phase shift at the time of transmission, random/cyclical phase of the combiner.

3. Scheduling of sufficiently large bandwidth, where the $\mathrm{SINR}_{\Delta}$ averages out due to frequency selective channel.

While the first approach always aims to keep the CQI mismatch positive, the two other approaches aim at set$\operatorname{ting} E\left(\operatorname{SINR}_{\Delta}\right)=0$.

Figure 9 shows the impact of BPSK cyclical phase shift per $\mathrm{PRB}$ on the CQI mismatch. A single frequency chunk of six PRBs has been scheduled in a round-robin manner. It can be seen significant that the cyclical phase shift efficiently averages out the above-mentioned CQI mismatch. While the LTE standard allows the phase shift per PRB, it might negatively impact the reliability of the dedicated channel estimation.

Figure 10 shows the average throughputs as a function of simulated TTIs per user drop. Again a single frequency chunk of six PRBs is being scheduled. The impact of OLLA correcting the CQI mismatch is visible. While the cyclical phase shift improves the performance of the link with a small amount of scheduled TTIs, after OLLA corrects the offset, the system without the cyclical phase shift performs better. In the case that the OLPA mechanism is applied, the performance of the link is significantly improved. The OLPA mechanism triggers the BPSK change of phase combiners $\theta_{2}$ between two transmission points across all scheduled PRBs. In this way, the transmission is kept coherent most of the time.

Figure 11 shows the impact of allocated bandwidth on the CQI mismatch. The CQI mismatch decreases with the scheduled bandwidth, though not as much as with CPS. Moreover, scheduling of $24 \mathrm{PRBs}$ to a SU is very rare.

\section{System-level CoMP simulation results}

For the evaluation of the network-level downlink performance of the LTE-Advanced system, we simulate 19 sites, each having 3 sectors as illustrated in Figure 5. In Scenario 3 , four RRHs are randomly located in the geographical area of each sector of a site. All the transmit points located in one site are assumed to be connected to the eNB with fiber connection. In these simulations, UEs are allowed to connect to center site points only, and points located in the rest of the sites are considered as interfering points. This is done to achieve a realistic UE placement so that the examined UEs are surrounded by interfering points,

Table 6 Non-coherent JT CoMP network performance in HetNet Scenario 3, Configuration 1 with different CQI feedback options

\begin{tabular}{lll}
\hline & $\begin{array}{l}\text { Average } \\
\text { (bps/Hz/point) }\end{array}$ & $\begin{array}{l}\text { Coverage } \\
\text { (bps/Hz/UE) }\end{array}$ \\
\hline SU-MIMO: $\mathrm{CQ}_{k, 1}^{\text {Rel } 10}$ & $1.848(0 \%)$ & $0.0367(0 \%)$ \\
$\mathrm{JT}: \mathrm{CQ}_{k}^{\mathrm{J}, \text { aggr. }}, \mathrm{CQI}_{k, 1}^{\text {Rel } 10}$ & $1.830(-1.0 \%)$ & $0.0406(10.6 \%)$ \\
$\mathrm{JT}: \mathrm{CQ}_{k, 1}^{\mathrm{DPS}}, \mathrm{CQ}_{k, 2}^{\mathrm{DPS}}$ & $1.828(-1.1 \%)$ & $0.0390(6.3 \%)$ \\
$\mathrm{JT}: \mathrm{CQ}_{k, 1}^{\mathrm{DPB}}, \mathrm{CQI}_{k, 2}^{\mathrm{DPB}}$ & $1.820(-1.5 \%)$ & $0.0336(-8.4 \%)$ \\
$\mathrm{JT}:\left.\mathrm{CQ}\right|_{k, 1} ^{\mathrm{DPB}}, \mathrm{CQ}_{k, 2}^{\mathrm{DPS}}$ & $1.819(-1.6 \%)$ & $0.0396(7.9 \%)$ \\
$\mathrm{JT}:\left.\mathrm{CQ}\right|_{k, 1} ^{\mathrm{DPB}}, \mathrm{CQ}_{k, 1}^{\mathrm{Rel} 10}, \mathrm{CQ}_{k, 2}^{\mathrm{DPB}}$ & $1.817(-1.7 \%)$ & $0.0389(6.0 \%)$ \\
\hline
\end{tabular}


Table 7 Non-coherent JT CoMP network performance in HetNet Scenario 3, Configuration 4b with different CQI feedback options

\begin{tabular}{|c|c|c|}
\hline & $\begin{array}{l}\text { Average } \\
\text { (bps/Hz/point) }\end{array}$ & $\begin{array}{l}\text { Coverage } \\
\text { (bps/Hz/UE) }\end{array}$ \\
\hline SU-MIMO: CQI Rel 10 & $2.387(0 \%)$ & $0.0627(0 \%)$ \\
\hline JT: $\left.C Q\right|_{k} ^{J T, \text { aggr. }},\left.C_{k}\right|_{k, 1} ^{R e l} 10$ & $2.386(-0.0 \%)$ & $0.0712(13.6 \%)$ \\
\hline $\mathrm{JT}: \mathrm{CQI}_{k, 1}^{\mathrm{DPS}}, \mathrm{CQI}_{k, 2}^{\mathrm{DPS}}$ & $2.378(-0.4 \%)$ & $0.0651(3.8 \%)$ \\
\hline $\mathrm{JT}: \mathrm{CQ}_{k, 1}^{\mathrm{DPB}}, \mathrm{CQ}_{k, 2}^{\mathrm{DPB}}$ & $2.364(-1.0 \%)$ & $0.0606(-3.3 \%)$ \\
\hline $\mathrm{JT}: \mathrm{CQI}_{k, 1}^{\mathrm{DPB}}, \mathrm{CQI}_{k, 2}^{\mathrm{DPS}}$ & $2.371(-0.7 \%)$ & $0.0682(8.8 \%)$ \\
\hline $\mathrm{JT}: \mathrm{CQI}_{k, 1}^{\mathrm{DPB}}, \mathrm{CQ}_{\mathrm{k}, 1}^{\mathrm{Rel}} 10, \mathrm{CQ}_{k, 2}^{\mathrm{DPB}}$ & $2.368(-0.8 \%)$ & $0.0676(7.8 \%)$ \\
\hline
\end{tabular}

which is the case in real networks. Interfering points are transmitting using random ranks and PMIs.

Two different UE dropping methods are used, uniform UE dropping (Configuration 1) and clustered dropping (Configuration 4b). After the UE is dropped, it selects its serving point. If the serving point is not located in the center site area, the UE is killed and a new UE is dropped. This is done until we have achieved the total number of UEs. All the points and UEs have two crosspolarized transmit antenna elements. Simulation flow consists of several simulation drops, where each drop has randomly generated UE positions. The simulation parameters follow 3GPP specification [30], while the UE dropping and the antenna radiation pattern are specified in [18]. In Table 4, we list the essential parameters and their values. All transmit points and UEs have two cross-polarized antenna elements, thus we simulate $2 \times 2$ MIMO.

In the following, the performance of JT and DPS CoMP is analyzed at system-level. Normal operation in the simulations is single-cell SU transmission. The selection of the CoMP reporting UEs is based on an average signal level of the serving point and the strongest interferer. CoMP is enabled to such cell-edge users that experience an average signal level difference between serving point and strongest interferer of less than $6 \mathrm{~dB}$. We have utilized OLLA operation per UE, and for each UE the eNB updates single OLLA value regardless of the transmission mode used. The major difference between the link-level studies presented in Section 4.5.2 and the system-level results presented in this section is the OLLA operation and the dynamic switching between the fallback single point mode and CoMP mode. For JT CoMP, the performance of different CQI options and the phase combiner feedback are shown in Sections 5.1 and 5.2, respectively. In Section 5.3, we present a comparison of DPS and JT with different handover margins. The handover margin is described in [30] and it is used as a threshold to avoid repetitive UE handovers between cells. In the simulated network operation, the serving point selection is biased by the handover margin such that the serving point is a random selection among points that have average signal strength within the handover margin compared to the strongest point.

\subsection{Non-coherent JT performance with different CQI options}

Non-coherent JT CoMP is simulated at system-level to see the effect of the different CoMP CQI alternatives described in Table 5. Simulation results are shown in Tables 6 and 7 for HetNet Scenario 3 Configurations 1 and $4 \mathrm{~b}$, respectively. Average transmit point spectral efficiency is defined as the average transmit point downlink throughput divided by the system bandwidth. The coverage is defined as the 5th percentile UE spectral efficiency that is the cell-edge user throughput divided by the system bandwidth.

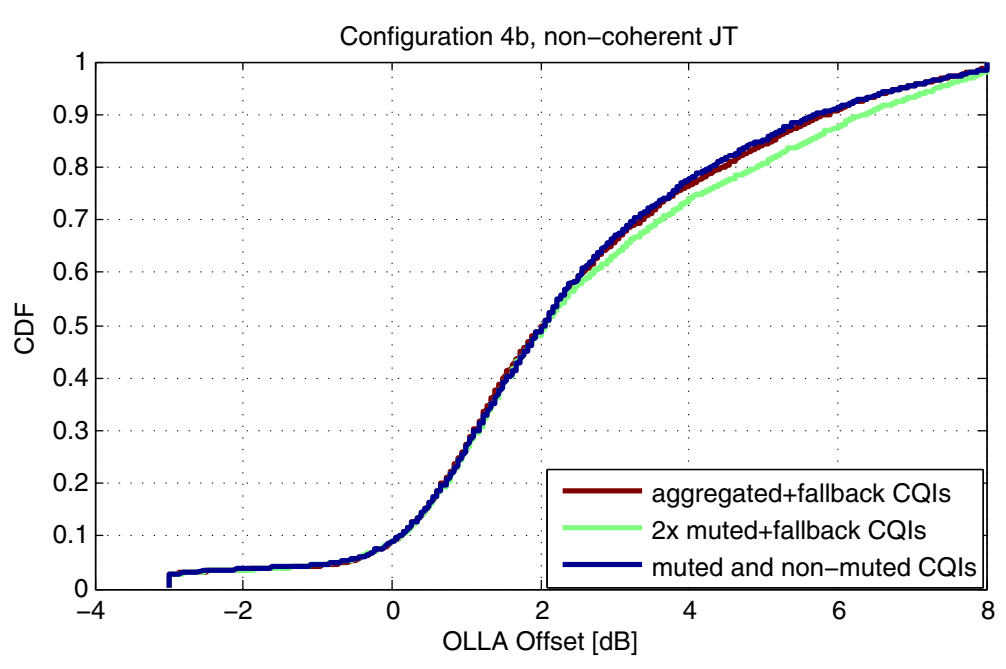

Figure 12 Cumulative density function of OLLA offset with different CQI feedback hypothesis. 
Table 8 Coherent JT network performance in HetNet

\begin{tabular}{|c|c|c|}
\hline & $\begin{array}{l}\text { Average } \\
\text { (bps/Hz/point) }\end{array}$ & $\begin{array}{l}\text { Coverage } \\
\text { (bps/Hz/UE) }\end{array}$ \\
\hline SU-MIMO: CQI Rel 10 & $1.848(0 \%)$ & $0.0367(0 \%)$ \\
\hline Non-coherent JT: CQI ${ }_{k}^{J, \text { aggr. }}$ & $1.830(-1.0 \%)$ & $0.0406(10.6 \%)$ \\
\hline JT with 1 bit combiner: CQI ${ }_{k}^{J T, a g g r .}$ & $1.848(0 \%)$ & $0.0428(16.6 \%)$ \\
\hline JT with 2bit combiner: $\mathrm{CQ}_{k}^{J T}$, aggr. & $1.856(0.4 \%)$ & $0.0433(18.0 \%)$ \\
\hline JT with 4bit combiner: $\mathrm{CQI}_{k}^{J T, \text { aggr. }}$ & $1.858(0.5 \%)$ & $0.0438(19.3 \%)$ \\
\hline
\end{tabular}

The average transmit point spectral efficiencies of JT with different CQI assumptions are similar to Release 10 SU-MIMO baseline. The minor performance degradation observed when CoMP is enabled is natural as the normal operation in the cell is single-cell operation and CoMP is performed mainly to cell-edge users. Overall, the best coverage gain is achieved with JT CoMP and aggregated CQI in both scenario configurations. Muted CQIs $\left(\mathrm{CQI}^{D P B}\right)$ without correct fallback CQI shows the worst performance due the approximated fallback CQI in both configurations. Interestingly, the two CQI feedback options, where one CQI is a non-muted CQI and the other CQI is the muted CQI, perform better than the feedback option having three CQIs, i.e., two muted CQIs with the additional fallback CQI. It may be noted that this is not in line with the link-level results presented in Section 4.5.2, where the sum of two muted CQIs was shown to have the best performance. Note that in the link-level simulations the OLLA process was scheduled band specific (roundrobin scheduling) and no dynamic switching between fallback and JT CoMP was allowed. With PF scheduling utilized here, different frequency sub-band resources can be assigned to users on a TTI basis. Thus, in the case of frequency selective channel, the CQI mismatch $\mathrm{SINR}_{\Delta}$ may vary according to results shown in Figure 9 as much as $13 \mathrm{~dB}$ between frequency sub-bands within one TTI. In system-level simulations, the single wideband OLLA process used both for JT CoMP as well as fallback operation works better if the estimated JT CQI is more pessimistic. The sum of two CQI ${ }^{\mathrm{DPS}}$ or the sum of a CQI ${ }^{\mathrm{DPS}}$ and a $\mathrm{CQI}^{\mathrm{DPB}}$ gives a more pessimistic estimate of the $\mathrm{CQI}^{\mathrm{IT}}$

Table 9 Coherent JT network performance in HetNet Scenario 3 Configuration $4 \mathrm{~b}, \mathrm{CQ}_{k}^{\mathrm{JT} \text {, aggr. }}, \mathrm{CQ}_{k, 1}^{\mathrm{Rel}} 10$

\begin{tabular}{|c|c|c|}
\hline & $\begin{array}{l}\text { Average } \\
\text { (bps/Hz/point) }\end{array}$ & $\begin{array}{l}\text { Coverage } \\
\text { (bps/Hz/UE) }\end{array}$ \\
\hline SU-MIMO: CQI Rel 10 & $2.387(0 \%)$ & $0.0627(0 \%)$ \\
\hline Non-coherent JT: $\mathrm{CQ}_{k}^{J T, \text { aggr. }}$ & $2.386(-0.0 \%)$ & $0.0712(13.6 \%)$ \\
\hline JT with 1 bit combiner: $\mathrm{CQI}_{k}^{J \mathrm{~T}, \text { aggr. }}$ & $2.415(1.2 \%)$ & $0.0737(17.5 \%)$ \\
\hline JT with 2bit combiner: $\mathrm{CQI}_{k}^{\mathrm{JT}, \text { aggr. }}$ & $2.428(1.7 \%)$ & $0.0739(17.9 \%)$ \\
\hline JT with 4bit combiner: CQI ${ }_{k}^{J, a g g r .}$ & $2.431(1.8 \%)$ & $0.0749(19.5 \%)$ \\
\hline
\end{tabular}

than the sum of two $\mathrm{CQI}^{\mathrm{DPB}}$. The impact of an overly optimistic CQI estimate can be seen in Figure 12, where a higher OLLA backoff for two CQI ${ }^{\mathrm{PDB}}$ is observed. In contrast, the more pessimistic approach shows similar OLLA backoff as aggregated CQI, especially in Configuration 4b.

\subsection{Coherent JT performance with quantized phase combiner}

System-level performance results of the phase combiner with different quantizations are shown in Tables 8 and 9 for HetNet Scenario 3 Configurations 1 and $4 \mathrm{~b}$, respectively. We used aggregated CQI (CQI ${ }^{T T, a g g r}$. $)$ since the aggregated CQI reflects the coherence gain estimated at the UE. Measurement error and delays are modeled to the phase combiner in the same way as to the other feedback. In the case of single-stream transmission, one phase combiner is needed but in the case that the UE reports rank 2, phase combiner per layer is assumed to be signaled. As in the previous case, the average transmit point spectral efficiencies are close to each other and only coverage gains are observed. Phase combiner gives a maximum of $7.9 \%$ coverage gain over the non-coherent $\mathrm{JT}$ in the case of Configuration 1 when 4-bits are used for the phase quantization. Based on these simulation results, simple 1-bit quantization captures the major part of the phase combiner gains and it seems to be a balanced compromise between the overhead and performance. However, one should note that phase combiner only attempts to improve the JT CoMP scheme and it has no use in the case of DPS or CS/CB CoMP.

\subsection{DPS versus JT CoMP and the effect of handover margin}

In addition to JT CoMP, other CoMP schemes are important in the LTE-Advanced evolution. In Tables 10 and 11, the performance of DPS CoMP and JT CoMP is shown with different handover margins (HO). The handover margin biases the transmit point selection in the simulation modeling, i.e., any of the potential serving points providing the strongest links within the margin according to the UE's measurements, may become the

Table 10 DPS and JT CoMP network performance in HetNet Scenario 3 Configuration 1 with different handover margins

\begin{tabular}{|c|c|c|}
\hline & $\begin{array}{l}\text { Average } \\
\text { (bps/Hz/point) }\end{array}$ & $\begin{array}{l}\text { Coverage } \\
\text { (bps/Hz/UE) }\end{array}$ \\
\hline SU-MIMO: CQI Rel $10, \mathrm{HO}=0 \mathrm{~dB}$ & $1.848(0 \%)$ & $0.0367(0 \%)$ \\
\hline 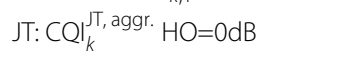 & $1.830(-1.0 \%)$ & $0.0406(10.6 \%)$ \\
\hline DPS: $\mathrm{CQ}_{k, 1}^{\mathrm{DPS}}, \mathrm{CQ}_{k, 2}^{\mathrm{DPS}}, \mathrm{HO}=0 \mathrm{~dB}$ & $1.821(-1.5 \%)$ & $0.0426(16.1 \%)$ \\
\hline SU-MIMO: CQI Rel $10, \mathrm{HO}=3 \mathrm{~dB}$ & $1.830(-1.0 \%)$ & $0.0292(-20.4 \%)$ \\
\hline$J \mathrm{~T}: \mathrm{CQ}_{k}^{\mathrm{J}, \text { aggr. }} \mathrm{HO}=3 \mathrm{~dB}$ & $1.812(-1.9 \%)$ & $0.0355(-3.3 \%)$ \\
\hline DPS: $\mathrm{CQ}_{k, 1}^{\mathrm{DPS}}, \mathrm{CQI}_{k, 2}^{\mathrm{DPS}}, \mathrm{HO}=3 \mathrm{~dB}$ & $1.814(-1.8 \%)$ & $0.0374(1.9 \%)$ \\
\hline
\end{tabular}


Table 11 DPS and JT CoMP performance in HetNet Scenario 3 Configuration $\mathbf{4 b}$ with different handover margins

\begin{tabular}{|c|c|c|}
\hline & $\begin{array}{l}\text { Average } \\
\text { (bps/Hz/point) }\end{array}$ & $\begin{array}{l}\text { Coverage } \\
\text { (bps/Hz/UE) }\end{array}$ \\
\hline SU-MIMO: $\mathrm{CQ}_{k, 1}^{\mathrm{Rel}}{ }^{10}, \mathrm{HO}=0 \mathrm{~dB}$ & $2.387(0 \%)$ & $0.0627(0 \%)$ \\
\hline $\mathrm{JT}: \mathrm{CQI}_{k}^{\mathrm{JT}, \text { aggr. }} \mathrm{HO}=0 \mathrm{~dB}$ & $2.386(-0.0 \%)$ & $0.0712(13.6 \%)$ \\
\hline DPS: $\mathrm{CQI}_{k, 1}^{\mathrm{DPS}}, \mathrm{CQ}_{k, 2}^{\mathrm{DPS}}, \mathrm{HO}=0 \mathrm{~dB}$ & $2.369(-0.6 \%)$ & $0.0684(9.1 \%)$ \\
\hline SU-MIMO: $\mathrm{CQI}_{k, 1}^{\mathrm{Rel}} 10, \mathrm{HO}=3 \mathrm{~dB}$ & $2.375(-0.5 \%)$ & $0.0508(-19.0 \%)$ \\
\hline $\mathrm{JT}: \mathrm{CQI}_{k}^{J \mathrm{~T}, \text { aggr. }} \mathrm{HO}=3 \mathrm{~dB}$ & $2.376(-0.5 \%)$ & $0.0641(2.2 \%)$ \\
\hline DPS: $\left.C Q\right|_{k, 1} ^{\text {DPS }}, \mathrm{CQI}_{k, 2}^{\mathrm{DPS}}, \mathrm{HO}=3 \mathrm{~dB}$ & $2.360(-1.1 \%)$ & $0.0641(2.2 \%)$ \\
\hline
\end{tabular}

serving point. With $0 \mathrm{~dB}$ handover margin the DPS CoMP provides approximately $1 \%$ decrease in average transmit point spectral efficiency compared to the Release $10 \mathrm{SU}$ MIMO baseline and over 16 and 9\% coverage gains for the simulated CoMP HetNet scenario 3 configurations 1 and $4 \mathrm{~b}$, respectively. The JT CoMP provides similar average spectral efficiency as baseline, while the coverage gains over the baseline are 11 and 14\% for HetNet Scenario 3 Configuration 1 and $4 \mathrm{~b}$, respectively.

Based on these results, we conclude that DPS CoMP can outperform JT CoMP in Configuration 1, however, in Configuration $4 \mathrm{~b}$ the situation changes. Overall, the gains between DPS and JT CoMP schemes are quite similar. In terms of the UE signal quality, JT CoMP is superior to the DPS as shown in Figure 13, where the the CoMP reporting UE's SINRs are compared. However, the JT CoMP SINR gain comes at the cost of using the resources from two different points. Therefore, in terms of system performance, the DPS CoMP can be a more efficient scheme than the JT CoMP.

When comparing the performance shown in Tables 10 and 11, it can be seen that with higher handover margins, overall performance degrades in both baseline and CoMP cases. For the SU-MIMO baseline, the point that is selected within the handover margin remains the serving point. Conversely, for DPS, the performance is partly recovered as the change of the transmission point is possible, thereby boosting CoMP performance relative to the baseline. These results show that there are substantial performance increases in CoMP gains for both JT and DPS CoMP. In the case of JT COMP, the 5th percentile throughput gain is roughly doubled, and in the case of DPS CoMP, the coverage gain of Configuration $4 \mathrm{~b}$ increases from 9 to $26 \%$. These simulation results indicate that CoMP is providing the highest gains over the baseline Release 10 system when handover cannot be performed in an optimal way. Thus, CoMP and especially DPS can be seen as a scheme to aid the mobility issues in real networks. This is an interesting and important practical finding of this study.

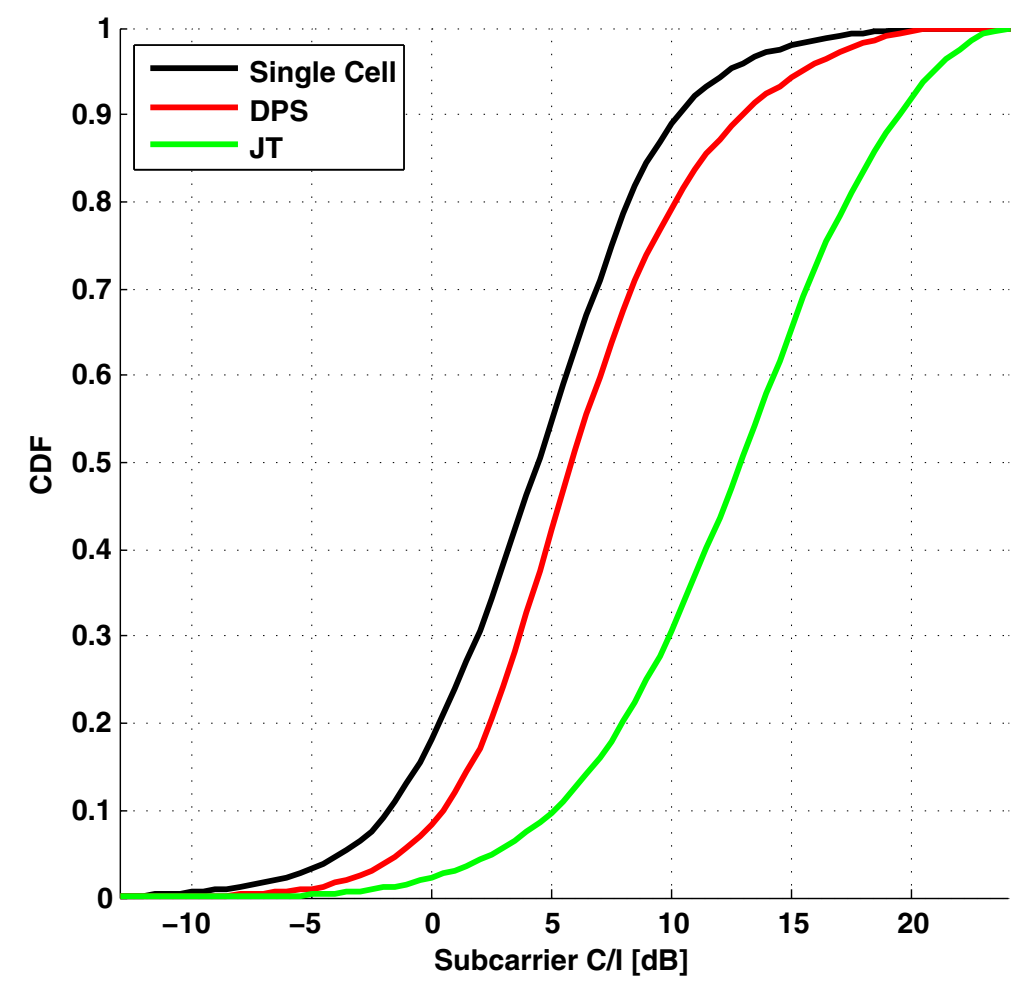

Figure 13 Cumulative density function of SINR with single-cell (point) transmission and two different multi-point schemes. 


\section{Conclusions}

In this article, we have addressed the problem of the feedback design and studied the associated link-level performance and the realistic system-level performance of CoMP in LTE-Advanced. We have studied practical finiterate CSI feedback and CoMP feedback design, namely PMI and CQI feedback, for different CoMP modes, and also evaluated the associated performance with both linklevel and system-level simulations. The realistic systemlevel evaluations of LTE-Advanced CoMP were performed for different CoMP modes and for different practical deployment scenarios. These simulation results indicate that CoMP can provide considerable cell-edge gains over the baseline Release 10 system with realistic UE feedback. The results that are obtained and reported in this study also indicate that the nature of the deployment scenario has a clear impact on the relative performance of JT and DPS type CoMP schemes. Relatively simple DPS schemes can outperform JT schemes in heterogeneous networks when the user distribution is not uniform but concentrated around the coverage area of the RRHs. When studying the CoMP schemes under biased handover conditions, it was observed that the DPS CoMP scheme can clearly aid in the mobility management of real networks. This is a very important practical benefit, in addition to improved cell edge performance, in cellular mobile radio systems.

\section{Competing interests}

The authors declare that, they have no competing interests.

\section{Author details \\ ${ }^{1}$ Renesas Mobile Europe Ltd., Porkkalankatu 24, 00180 Helsinki, Finland. ${ }^{2}$ Department of Communications Engineering, Tampere University of Technology, Korkeakoulunkatu 1, FI-33720 Tampere, Finland. ${ }^{3}$ Department of Signal Processing and Acoustics, Aalto University, P.O. Box 13000, Fl-00076 Aalto, Finland.}

Received: 22 June 2012 Accepted: 16 October 2012 Published: 27 November 2012

\section{References}

1. 3rd Generation Partnership Project; TSG RAN: (E-UTRA); 3GPP TS 36.211 version 10.0.0 Physical channels and modulation (Release 10) (2010). http://www.3gpp.org/

2. 3rd Generation Partnership Project; TSG RAN: (E-UTRA); 3GPP TS 36.213 version 10.0.1 Physical layer procedures (Release 10) (2010). http://www. 3gpp.org/

3. 3rd Generation Partnership Project; TSG RAN: (E-UTRA); 3GPP TS 36.213 version 8.5.0 Physical layer procedures (Release 8) (2008). http://www. 3gpp.org/

4. 3rd Generation Partnership Project; TSG RAN: (E-UTRA); 3GPP TS 36.213 version 11.0.0 Physical layer procedures (Release 11) (2012). http://www. 3gpp.org/

5. YH Nam, L Liu, Y Wang, C Zhang, J Cho, JK Han, in Proc. IEEE ICASSP. Cooperative communication technologies for LTE-Advanced (Dallas, TX 2010), pp. 5610-5613

6. C Khirallah, D Vukobratovic, J Thompson, in Proc. IEEE WSA. On energy efficiency of joint transmission coordinated multi-point in LTE-Advanced (Dresden, Germany, 2012), pp. 54-61

7. L Qian, Y Yang, F Shu, W Gang, in Proc. IEEE CHINACOM 5. Coordinated beamforming in downlink CoMP transmission system (Beijing, China, 2010), pp. 1-5
8. Q Cui, S Yang, Y Xu, X Tao, B Liu, in Proc. IEEE VTC 74. An effective inter-cell interference coordination scheme for downlink CoMP in LTE-A systems (San Francisco, CA, 2011), pp. 1-5

9. HW Je, H Lee, K Kwak, S Choi, YJx Hong, YJ Clerckx, in Proc. IEEE GLOBECOM. Long-term channel information-based CoMP beamforming in LTE-Advanced systems (TX, Houston, 2011), pp. 1-6

10. ZTan, W Zhou, W Chen, S Chen, Y Xu, in Proc. IEEE ICCTA 17. A dynamic cell selection scheme based on multi-object for COMP DL in LTE-A (Beijing, China, 2011), pp. 248-252

11. S Gong, P Zhu, X Meng, X You, in Proc. IEEE WCSP. Wideband dynamic base station selection for downlink coordinated multipoint system (Nanjing, China, 2011), pp. 1-5

12. Y Gao, Y Li, H Yu, S Gao, in Proc. IEEE ICCSN 5. Performance of dynamic CoMP cell selection in 3GPP LTE system level simulation (Xi'an, China, 2011), pp. 210-213

13. $Y$ Gao, Y Li, H Yu, S Gao, in Proc. IEEE URKE. Performance analysis of dynamic CoMP cell selection in LTE-advanced heterogeneous networks scenario, (Bali, 2011), pp. 173-176

14. M Sawahashi, Y Kishiyama, A Morimoto, D Nishikawa, M Tanno, Coordinated multipoint, transmission/reception techniques for LTE-advanced [Coordinated and Distributed MIMO]. IEEE Trans. Wirel. Commun. 17(3), 26-34 (2010)

15. H D Lee, B Seo, E Clerckx, D Hardouin, S Mazzarese, K Nagata, Sayana, Coordinated multipoint transmission and reception in LTE-advanced: deployment scenarios and operational challenges. IEEE Commun. Mag. $\mathbf{5 0}(2), 148-155$ (2012)

16. XXu X Tao, Q Cui, An overview of cooperative communications. IEEE Commun. Mag. 50(6), 65-71 (2012)

17. R Irmer, H Droste, P Marsch, M Grieger, G Fettweis, S Brueck, HP Mayer, L Thiele, $V$ Jungnickel, Coordinated multipoint: concepts, performance, and field trial results. IEEE Commun. Mag. 49(2), 102-111 (2011)

18. 3rd Generation Partnership Project; TSG RAN: (E-UTRA); 3GPP TR 36.814 version 9.0.0 Evolved Universal Terrestrial Radio Access (E-UTRA); Further advancements for E-UTRA physical layer aspects (Release 11) (2010). http://www.3gpp.org/

19. KK Mukkavilli, A Sabharwal, E Erkip, B Aazhang, On beamforming with finite rate feedback in multi-antenna systems. IEEE Trans. Inf. Theory. 49(10), 2562-2579 (2003)

20. DJ Love, RW Heath, T Strohmer, Grassmannian beamforming for multiple-input multiple-output wireless systems. IEEE Trans. Inf. Theory. 49(10), 2735-2747 (2003)

21. HL Määttänen, K Schober, O Tirkkonen, R Wichman, Precoder partitioning in closed-loop MIMO systems. IEEE Trans. Wirel. Commun. 8(8), 3910-3914 (2009)

22. T Shuang, T Koivisto, HL Määttänen, K Pietikäinen, T Roman, M Enescu, in Proc. IEEE VTC, vol. 75. Desing and evaluation of LTE-Advanced double codebook (Yokohama, Japan, 2012), pp. 1-5

23. HL Määttänen, K Schober, O Tirkkonen, R Wichman, in Proc. WPMC 11. Tradeoff between grassmannian and orthogonalizing precoding with linear receiver, (Oulu, Finland, 2008), pp. 1-5

24. HL Määttänen, T Koivisto, M Enescu, O Tirkkonen, CSI feedback for dynamic switching between single user and multiuser MIMO. Wirel Personal Commun. 64(1), 33-49 (2012)

25. HL Määttänen, T Huovinen, T Koivisto, M Enescu, O Tirkkonen, M Valkama, in Proc. IEEE VTC 73. Performance evaluations for multiuser CQI enhancements for LTE-Advanced (Budapest, Hungary, 2011). pp. 1-5

26. K Pedersen, G Monghal, I Kovacs, T Kolding, A Pokhariyal, F Frederiksen, P Mogensen, in Proc. IEEE VTC 66. Frequency domain scheduling for OFDMA with limited and noisy channel feedback, (Baltimore, MD, 2007), pp. 1792-1796

27. J Han, P Wang, F Liu, Y Zhu, Block Diagonal Precoding Based Power Allocation for Coordinated Multi-Point Transmission. Journal of Commun. 6(7), 524-528 (2011)

28. 3rd Generation Partnership Project; TSG RAN: Evolved Universal Terrestrial Radio Access (E-UTRA); X2 General Aspects and Principles) (2008). http:// www.3gpp.org/

29. 3rd Generation Partnership Project; R1-071804: Reply LS to R3-070527/R1-071242 on Backhaul (X2 interface) Delay (2007). http:// www.3gpp.org/ 
30. 3rd Generation Partnership Project; TSG RAN: (E-UTRA): 3GPP TR 36.819 version 11.1.0 Coordinated multi-point operation for LTE physical layer aspects (Release 11) (2011). http://www.3gpp.org/

31. B Clerckx, Y Kim, H Lee, J Cho, J Lee, in Proc. IEEE MWSCAS 54. Coordinated multi-point transmission in heterogeneous networks: a distributed antenna system approach (Seoul, South Korea, 2011), pp. 1-4

32. YX Cheng, Y Lau, Y Long, A scalable limited feedback design for network MIMO using per-cell product codebook. IEEE Trans. Wirel. Commun. 9(10), 3093-3099 (2010)

33. RA Pitaval, O Tirkkonen, in Proc. IEEE VTC, vol. 75. Incorporating stiefel geometry in codebook design and selection for improved base station cooperation (Yokohama, Japan, 2012), pp. 1-5

34. S Nonchev, J Venäläinen, M Valkama, M Kuusela, in Proc. ICT. New frequency domain packet scheduling schemes for UTRAN LTE downlink, (Sweden, Stockholm, 2008), pp. 1-5

35. K Schober, R Wichman, T Roman, in Proc. CISS 46. Layer arrangement for single-user coordinated multi-point transmission, (Princeton, NJ, 2012), pp. 1-5

36. ITU: Guidelines for evaluation of radio technologies for IMT-Advanced. TS ITU-R,M.2135 (2008). http://www.itu.int/publ/R-REP-M.2135-2008/en

doi:10.1186/1687-6180-2012-247

Cite this article as: Määttänen et al: System-level performance of LTEAdvanced with joint transmission and dynamic point selection schemes. EURASIP Journal on Advances in Signal Processing 2012 2012:247.

\section{Submit your manuscript to a SpringerOpen ${ }^{\mathcal{O}}$ journal and benefit from:}

- Convenient online submission

- Rigorous peer review

- Immediate publication on acceptance

- Open access: articles freely available online

- High visibility within the field

- Retaining the copyright to your article

Submit your next manuscript at $\boldsymbol{\nabla}$ springeropen.com 